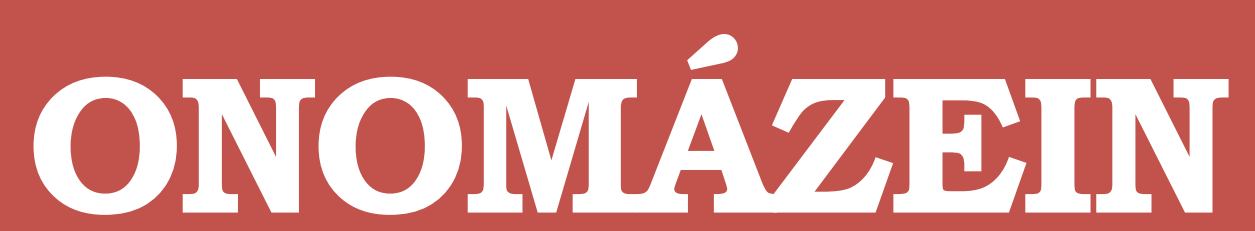

Revista de lingüística, filología y traducción
PONTIFICIA UNIVERSIDAD

\title{
Método de transcripción del japonés al español: sonidos vocálicos, semivocálicos y consonánticos
}

Method of transcription from Japanese to Spanish:

vocalic, semi-vocalic and consonant sounds

\section{Rafael Fernández Mata \\ Universidad de Córdoba \\ España}

\section{(C) $($ (i) $\ominus$}

Rafael Fernández Mata: Departamento de Ciencias del Lenguaje. Universidad de Córdoba, España. | Correo electrónico: rafaelfernandezmata@gmail.com 


\section{Resumen}

Nunca antes se había elaborado un método de transcripción en español con el cual poder representar de manera rigurosa y unitaria las voces de origen japonés. En este artículo se repasa brevemente el sistema de escritura de la lengua japonesa actual y se ofrecen las claves necesarias para proceder a la transcripción de los sonidos vocálicos, semivocálicos y consonánticos de la lengua originaria, el japonés, a la lengua de destino, el español.

Palabras clave: sonidos vocálicos del japonés; sonidos semivocálicos del japonés; sonidos consonánticos del japonés; canyi; jiragana; catacana.

\section{Abstract}

For the first time ever, a method of transcription from Japanese to Spanish that represents accurately, and by unit, the loanwords from the Japanese language using the Spanish alphabet, is proposed. The writing system of contemporary Japanese is summarized and the necessary keys to transcribe the vocalic, semi-vocalic and consonant sounds of the source language, Japanese, to the target language, Spanish, are suggested.

Keywords: vocalic sounds from Japanese; semi-vocalic sounds from Japanese; consonant sounds from Japanese; kanji; hiragana; katakana. 


\section{Introducción}

\subsection{El sistema de escritura del japonés estándar}

En la actualidad, el sistema de escritura del japonés estándar, unánimemente reconocido como uno de los más complejos de todas las lenguas (Vance, 1987: 2; Seeley, 2000 [1991]: IX; Del Moral, 2002: 267; Labrune, 2012: 9) -lo cual contrasta enormemente con la sobriedad de su sistema fonológico-, se compone de cuatro tipos de signos gráficos: los canyis, los canas - formados por los silabarios jiragana y catacana- y, en menor medida, el alfabeto latino y los números arábigos (Vance, 1987: 2-3; Shibatani, 1990: 125; Japan Travel Bureau, 1991 [1989]: 12-13; Rodríguez-Izquierdo, 1991: 121; Akamatsu, 1997: 4-5; Matsuura y Porta, 2000: 30; Seeley, 2000 [1991]: 152-179; Ferres, 2001: 13-15; Unger, 2003 [1996]; Frellesvig, 2010: 157-183; Labrune, 2012: 8-9).

Los canyis (de 漢字 [kanz'i] 'letras o caracteres de [el grupo étnico o cultural] Han’) son ideogramas de origen chino que se usan generalmente para escribir en japonés moderno los morfemas léxicos de origen chino-japonés, esto es, la raíz de sustantivos, verbos, adjetivos y algunos adverbios está representada en canyi, mientras que la parte flexional se transcribe en cana (Vance, 1987: 2-3; Shibatani, 1990: 128; JTB, 1991 [1989]: 12-13; Rodríguez-Izquierdo, 1991: 121; Akamatsu, 1997: 4-5; Matsuura y Porta, 2000: 26; Seeley, 2000 [1991]: 152-179; Ferres, 2001: 12 y 15; Frellesvig, 2010: 157-183; Labrune, 2012: 11-12). No se tiene constancia del número exacto de canyis, aunque el Consejo de Ministros de Japón reelaboró en 1981 una lista compuesta por 1945 ideogramas con los canyis de uso normal, esto es, los que un adulto educado ha de conocer, si bien un adulto japonés está expuesto diariamente a unos 4000 canyis (Matsuura y Porta, 2000: 26; Ferres, 2001: 26-27; Labrune, 2012: 12).

La adopción de un sistema de escritura foráneo acarreó serios problemas para la representación gráfica. La parte invariable, el morfema léxico representado mediante un ideograma, no acarreó problemas de adaptación; el morfema gramatical, sí. La parte variable se transcribía usando canyis como fonogramas, esto es, como meros representantes de sonidos, sin significación inherente. Con el tiempo, se produjo la simplificación de estos elementos y dio lugar a los canas 一仮名, [kana], 'nombre provisional o no definitivo' - (Vance, 1987: 2-3; Shibatani, 1990: 126-127; JTB, 1991 [1989]: 18-19; Akamatsu, 1997: 4-5; Matsuura y Porta, 2000: 26-27; Seeley, 2000 [1991]: 59-80; Ferres, 2001: 13-15; Frellesvig, 2010: 158-159; Labrune, 2012: 8). Bajo la etiqueta genérica de cana se recogen los silabarios jiragana (平仮名, [h'ilagana], 'cana simple / informal') y catacana (片仮名, [katakana], 'cana incompleto'). El primero se utiliza

1 El sistema de transcripción fonético-fonológica empleado en este artículo es el mismo que desarrollamos en nuestra tesis inédita, Los japonesismos de la lengua española: Historia y transcripción. Todos los símbolos cuentan con sus explicaciones fonética y articulatoria, que podrán verse en sus respectivos epígrafes dentro de este artículo. 
principalmente para la inflexión de verbos, adjetivos, para partículas (elementos de relación pospuestos), interjecciones, conectores y algunos adverbios. A veces se usan para escribir morfemas léxicos que el escritor no quiere o no sabe cómo escribir, o bien porque el canyi ha quedado obsoleto. Por su parte, el catacana está generalmente reservado a la transcripción de préstamos recientes, para las palabras mimético-onomatopéyicas, nombres de plantas o animales, formas dialectales o jergales, e incluso, a veces, para palabras eruditas. Asimismo puede usarse para resaltar un elemento en una oración, bien para marcar ironía o para darle un tono más coloquial u oral (Vance, 1987: 3-4; Shibatani, 1990: 128; JTB, 1991 [1989]: 13; Matsuura y Porta, 2000: 26-27; Seeley, 2000 [1991]: 152-179; Ferres, 2001: 13-15; Del Moral, 2002: 267-268; Labrune, 2012: 10).

Mostramos, a continuación, una oración con la que reflejar gráficamente lo arriba expuesto:

\begin{tabular}{|c|c|}
\hline \multicolumn{2}{|c|}{$\begin{array}{l}\text { 田中さんは友達たちと喫茶店でコーヒーを飲んでいます。 } \\
\text { ‘El señor Tanaca está bebiendo café con unos amigos en la cafetería’ }\end{array}$} \\
\hline 田中[tanaka] & Lexema sustantivo: apellido japonés. Escrito en canyi. \\
\hline さん [san] & $\begin{array}{l}\text { Sufijo que se añade al nombre para indicar cortesía. } \\
\text { Escrito en jiragana. }\end{array}$ \\
\hline は [ща] & $\begin{array}{l}\text { Partícula pospuesta que marca la función de sujeto de la } \\
\text { oración. Escrita en jiragana. Cuando el cana は, /ha/, fun- } \\
\text { ciona como marca denotativa de sujeto se pronuncia [ua]. }\end{array}$ \\
\hline 友達 [tomodati] & Lexema sustantivo ('amigo/a’). Escrito en canyi. \\
\hline たち [tatfi] & Sufijo de plural. Escrito en jiragana. \\
\hline と [to] & $\begin{array}{l}\text { Partícula pospuesta que marca la función de complemento } \\
\text { circunstancial de compañía. Escrita en jiragana. }\end{array}$ \\
\hline 喫茶店 [kissaten] & Lexema sustantivo ('cafetería’). Escrito en canyi. \\
\hline \multirow[b]{2}{*}{ コーヒー [ko:h'is] } & $\begin{array}{l}\text { Partícula pospuesta que marca la función de complemento } \\
\text { circunstancial de lugar. Escrita en jiragana. }\end{array}$ \\
\hline & $\begin{array}{l}\text { Lexema sustantivo ('café’) procedente de la lengua inglesa } \\
\text { (<coffee). Escrito en catacana. }\end{array}$ \\
\hline を [o] & $\begin{array}{l}\text { Partícula pospuesta que marca la función de complemen- } \\
\text { to directo. Escrita en jiragana. Cuando el cana を,/uo/, fun- } \\
\text { ciona como marca denotativa de complemento directo se } \\
\text { pronuncia [o]. }\end{array}$ \\
\hline \multirow[b]{2}{*}{ んでいます[ndeimasu] } & Lexema verbal (‘beber’). Escrito en canyi. \\
\hline & $\begin{array}{l}\text { Parte flexional del verbo: indica presente continuo ('estar } \\
\text { + gerundio'). Escrito en jiragana. }\end{array}$ \\
\hline$\circ$ & $\begin{array}{l}\text { Signo gráfico de puntuación: indica punto (= .). Se escribe } \\
\text { así tanto en jiragana como en catacana. }\end{array}$ \\
\hline
\end{tabular}




\subsection{Los modelos extranjerizantes Hepburn y Kunrei}

Dos de los métodos más utilizados para la transcripción ortográfica del japonés al alfabeto latino surgieron a finales del siglo XIX, coincidiendo con la apertura de Japón al mundo. El primero, conocido como estilo Hepburn, llamado así por su inventor, el médico misionero norteamericano James Curtis Hepburn (1815-1911), fue adoptado por el Club de la Romanización (sociedad formada en Japón por extranjeros y japoneses con el fin de promover la escritura en alfabeto latino) en 1885, y por Hepburn, quien lo usó para la tercera edición de 1886 de su famoso diccionario japonés-inglés. El segundo método fue ideado por el físico japonés Tanakadate Aikitsu en 1885 y se le conoció como Nippon-shiki ('sistema japonés'). Este último evolucionó hacia el Kunrei-shiki ('sistema oficial' o ‘según las instrucciones') en 1937, cuando fue oficialmente adoptado por el gobierno de la época. En la actualidad conviven estos métodos de transcripción, con alguna modificación, sin embargo, el sistema Hepburn es el preferido en diccionarios, textos romanizados dirigidos a docentes y estudiantes de japonés como segunda lengua, y en la adaptación de nombres propios² (Rodríguez-Izquierdo, 1991: 124-125; Matsuura y Porta, 2000: 30-32; Seeley, 2000 [1991]: 138-151; Ferres, 2001: 16-17; Vance, 2008: 239-244; Gottlieb, 2010: 75-88)3.

El motivo de que el método Hepburn sea el favorito fuera de Japón se debe a que está enfocado en representar la fonética del japonés, esto es, existe una relación más estrecha entre grafema y sonido. En cambio, el sistema Kunrei queda enfocado hacia las reglas ortológicas de la lengua japonesa y es más sistemático y regular (JTB, 1991 [1989]: 21; Rodríguez-Izquierdo, 1991: 124-125 y 127; Matsuura y Porta, 2000: 27-32; Seeley, 2000 [1991]: 200; Ferres, 2001: 20-22; Tsujimura, 2007 [1996]: 6; Vance, 2008: 241-244; Frellesvig, 2010: 159; Labrune, 2012: 8-9). De esta forma, por ejemplo, el cana つ [tsu] en Hepburn se transcribiría como tsu, mientras que en Kunrei se utilizaría tu4.

El sistema Hepburn está basado en los grafemas de las vocales italianas y los grafemas de los sonidos consonánticos del inglés (Rodríguez-Izquierdo, 1991: 124; Matsuura y Porta,

2 Este es el motivo por el cual no aplicamos nuestro método de transcripción a las referencias bibliográficas empleadas en este artículo (nombres propios de autores o editoriales, por ejemplo).

3 De hecho, Matsuura y Porta (2000: 31) y Ferres (2001: 17) lo utilizan en sus manuales, que van dirigidos a hispanohablantes.

4 Apréciese cómo la primera transcripción (tsu), en Hepburn, refleja mejor la pronunciación del japonés. La segunda forma (tu), al contrario, se adecua al sistema ortológico del japonés; esto último está conectado con la forma en que los silabarios jiragana y catacana se disponen, ya que no siguen la distribución de nuestro alfabeto. Se comienza por las vocales $(a, i, u, e, 0)$, posteriormente por la línea del sonido [k] (ka, ki, ku, ke, ko), luego, la línea de la s (sa, shi, su, se, so), etc. Así, a un hablante nativo japonés no le importa que el kana $\supset$ se represente mediante tsu o mediante $t u$, ya que sabe que su pronunciación es asibilada ante /u/. 
2000: 30; Ferres, 2001: 16-17), de ahí el desajuste al tratar de aplicarlo a la transcripción de préstamos léxicos en español, puesto que no se adapta a determinados casos. En efecto, hemos detectado que algunas voces procedentes del japonés han sido transcritas en nuestra lengua mediante el método de transcripción Hepburn5, por lo que su forma gráfica responde a cánones grafo-fonológicos extraños a nuestro sistema ${ }^{6}$.

Nuestro cometido, pues, será desarrollar un sistema de transcripción del japonés al español que se adecue a los cánones españoles. Y, para conseguirlo, consideraremos los preceptos ortográficos de la Real Academia Española [RAE], en especial las recomendaciones aportadas por la RAE (2010: 621-622) sobre la hispanización de las transcripciones de voces procedentes de lenguas que no emplean el alfabeto latino.

\section{Nuestro método de transcripción}

Una de las características principales que asumiremos para nuestro método de transcripción ortográfica será su unidireccionalidad. No crearemos un modelo de romanización de la escritura japonesa para ser usado en manuales de enseñanza de japonés como lengua extranjera. Nuestro modelo nace como necesidad, tras comprobar que los préstamos léxicos de origen japonés presentan serias dificultades para ser transcritos en español, bien porque se desconoce la fonología del étimo — recuérdese al respecto que no existen trabajos sobre fonología japonesa en español-, bien porque se utiliza el sistema de transcripción Hepburn —que no se adapta a las reglas grafofonológicas del español-, o bien por la existencia de doble posibilidad de representación en español, lo que provoca numerosos dobletes gráficos y y dudas en su escritura, que no hacen más que contravenir el concepto de economía lingüística propuesto por Martinet (1974: 133-134). Este modelo de transcripción ha sido creado para representar solo palabras que forman parte del sistema léxico del español, central o periférico, y que proceden de la lengua japonesa.

5 El poder casi absoluto de este método de transcripción encuentra su causa en la hegemonía de la lengua inglesa sobre otros sistemas lingüísticos.

6 Por ejemplo, en el Diccionario Clave: diccionario de uso del español actual (2012), encontramos 36 voces transcritas con patrones gráficos extranjerizantes: aikido, aikidoka, banzai, butoh, dojo, geisha, ginkgo (gingko), haiku (haikai o hai-kai), ikebana, jiu-jitsu, kabuki, kaizen, karaoke, kárate (karate), karateca (karateka), keirin, kendo, ninja, otaku, reiki, sake, sashimi, sesshin, shabu shabu, shiatsu, shiso, sudoku, sushi, tamagotchi, tepanyaki (teppan-yaki), teriyaki, toyotismo, tsunami, yen, zazen y zen.

7 En la actualidad, por ejemplo, el Diccionario de la Real Academia Española (RAE, 2014, vigesimotercera edición) recoge los siguientes dobletes: caqui o kaki, catana o catán — para saber más sobre este japonesismo cfr. Fernández Mata (2015) -, haikai o hai-kai, haiku o haikú, kárate o karate, mikado o micado, kimono o quimono, samurái o samuray, soja o soya, tsunami o sunami, yudo o judo. 
Se compararán los sistemas fonético-fonológicos del japonés y del español ${ }^{8}$ con el objeto de relacionar los sonidos con mayor similitud y establecer un rango de categorías gráficas con que representarlos. Primarán los rasgos distintivos de la lengua de destino, por lo que obviaremos aquellas cualidades fonológicas que son pertinentes en la lengua japonesa, pero que no funcionan en lengua española. Al respecto, insistimos en que no pretendemos desarrollar un modelo de transcripción reversible. A pesar de esta irreversibilidad, los nombres propios y los topónimos, por ejemplo, pueden seguir las reglas de nuestro sistema de transcripción. Por tanto, las capitales 東京 [to:k'o:], 京都 [k'oxto] y 大阪 [orsaka], con vocales largas — no pertinentes en español-, se transcribirían como Toquio, Quioto y Osaca sin ningún problema.

Dividiremos el material que compone el sistema fonológico del japonés de acuerdo con la naturaleza de la raíz y el valor distribucional de sus unidades, como ya hicimos en el bloque de fonética y fonología de la lengua japonesa de nuestra tesis inédita, Los japonesismos de la lengua española: Historia y transcripción.

En definitiva, tras ofrecer unas pautas fundamentadas basadas en la sencillez y coherencia, pretendemos desarrollar un modelo de transcripción intuitivo, que permita a cualquier usuario, con un mínimo conocimiento de las reglas básicas de escritura española, adaptar un japonesismo dado a nuestro idioma sin necesidad de intrincadas normas y constantes excepciones.

\section{Unidades vocálicas del japonés}

Para la observación fonético-articulatoria de las realizaciones vocálicas del japonés, hemos tenido presentes cuatro obras claves: de un lado, el análisis funcionalista de los trabajos de Akamatsu (1997 y 2000); de otro, la visión de Vance (1987 y 2008). Tanto uno como otro se basan en el estudio de la Nihon Onsei Gakkai, esto es, la Sociedad de Fonética del Japón, la cual, mediante la utilización de rayos $X$, estableció un diagrama vocálico para el japonés, comparable con el que Abercrombie (1967: 151-162) dispuso para el inglés. La visión tradicional de

8 Hemos empleado obras de fonología española de corte funcional, generativistas, o bien de carácter ecléctico, ya que elegimos un proceder funcionalista con un eclecticismo moderado para realizar el análisis del sistema fonológico del japonés. El compendio descriptivo y unitario del inventario de fonemas de la lengua española que seguiremos contará con las 24 unidades propuestas por Alarcos (1986 [1965]), y consolidadas por Quilis (2006 [1993]), Hualde (2005) y RAE (2011). No obstante, como ya advertimos, tendremos en cuenta la visión de otros autores: Canellada y Kuhlmann (1987), Martínez Celdrán (1989 y 2000), D’Introno, Del Teso y Weston (1995), Hidalgo y Quilis (2004, 2012), Iribarren (2005), Gil (2007) y Martínez Celdrán y Fernández Planas (2007).

9 Los autores citan la página que han consultado de la Sociedad de Fonética del Japón, ed. (1976: 735): Onseigaku Daijiten, Toquio: Sanshusha. 
Abercrombie (1967) describía la articulación vocálica mediante la posición que adoptaba la lengua dentro de la cavidad bucal —como a principios del siglo XX hizo Navarro Tomás (2004 [1918]) para el español-, dando como resultado una descripción vocálica que se conoció como vocales cardinales.

\subsection{Descripción articulatoria}

Dentro del conjunto de sonidos vocálicos, describiremos, en primer lugar, dos sonidos, [i] y [e], que poseen dos características semejantes: por un lado, los labios no presentan abocinamiento y, por otro, se articulan en la zona anterior de la cavidad bucal. Para modular [i], la boca se mantiene levemente abierta, con la mandíbula inferior elevada; el ápice de la lengua se apoya en los incisivos inferiores mientras que el dorso se eleva hacia el paladar, adquiriendo la lengua una forma convexa (Akamatsu, 1997: 30; Vance, 2008: 53). La vocal /i/ se encuentra en palabras japonesas como 胃 [i] 'estómago', 島 [s'ima] 'isla', 港 [m'ĩnãto] 'puerto', 蟻 [al'i] ‘hormiga' (Akamatsu, 1997: 30). Aunque se asemeja bastante a nuestra [i], es ligeramente inferior y posterior a esta, es decir, el punto más alto del dorso lingual no se encuentra tan próximo a la zona alveolo-palatal (Akamatsu, 1997: 30).

En cuanto a [e], la boca mantiene una abertura mayor que en [i], con la mandíbula más baja. La lengua se encuentra a una distancia mayor entre el dorso y el paladar, por lo que esta realización permanece en una zona intermedia del espacio oral (Akamatsu, 1997: 32; Vance, 2008: 53). La vocal /e/ tiene lugar en palabras japonesas como 絵 [e] 'pintura, ilustración', 手 [te] 'mano', 切れ [k'ile] 'tela, trapo', 蛙 [kaelu] 'rana' (Akamatsu, 1997: 33). Es bastante similar a nuestra [e]: la [e] japonesa se sitúa entre nuestra [e] abierta y cerrada (Akamatsu, 1997: 3233; Vance, 2008: 53):

Por su parte, para la pronunciación de [a], la mandíbula inferior baja y la boca queda claramente abierta. Se articula en la parte central de la cavidad, hecho que, como analizaremos, no comparte con ninguna otra vocal. Además, en contraste con el resto de sonidos vocálicos, se caracteriza por que la lengua, cuyo ápice toca los incisivos inferiores y cuyo dorso se mantiene plano, se localiza en la parte más baja de la cavidad (Akamatsu, 1997: 34; Vance 2008: 54). La vocal /a/ se da en palabras japonesas como 蚊 [ka] 'mosquito', 赤 [aka] 'el rojo' (Akamatsu, 1997: 35). Según Akamatsu (1997: 34), es muy similar a la realización española.

En último lugar, al igual que las vocales anteriores [e]-[i], en la realización de las vocales [o] y [u] encontramos características comunes: de un lado la acción labial —sobre la que informaremos en párrafos siguientes - y, de otro, el lugar de articulación: zona posterior de la cavidad bucal. Para articular [o], la mandíbula baja hasta mantener la boca una abertura media. El posdorso lingual se eleva hacia el velo del paladar, pero se mantiene separado del mismo (Akamatsu, 1997: 33; Vance, 2008: 53). La vocal /o/ tiene lugar en palabras japonesas 
como 尾 [o] 'cola, rabo', 戸 [to] 'puerta', 角 [kado] 'esquina', 男 [otoko] 'hombre' (Akamatsu, 1997: 34). Al igual que la vocal media [e], [o] se encuentra a medio camino entre nuestra [o] abierta y cerrada, pero con muy poco redondeamiento labial: en este caso, solo las comisuras de la boca se aproximan horizontalmente de manera muy suave, apenas perceptible, sin que se produzca una fuerte protrusión labial (Akamatsu, 1997: 33; Vance, 2008: 54).

En lo que respecta a $[\boldsymbol{m}]$ — el alófono más extendido de /u/_, la mandíbula se eleva, manteniendo la boca una abertura mínima. La vocal/u/ tiene lugar en palabras japonesas como 鵜 [u] 'cormorán', 雲 [kumo] 'nube', 犬 [inu] 'perro' (Akamatsu, 1997: 32). El posdorso lingual se aproxima hacia el velo del paladar, pero, a diferencia de la articulación española que es más posterior, la japonesa es mucho más adelantada y baja que la española (Akamatsu, 1997: 31; Vance, 2008: 54-56); esto es, la parte posterior de la lengua no se eleva tanto hacia el velo del paladar, sino que se mantiene cercana a este y al paladar. Asimismo, no existe protrusión labial para este sonido (Ueda, 1977: 30; Rodríguez-Izquierdo, 1991: 122; Hara, 1994: 371; Kaneko, 2006: 51; Quilis, 2006 [1993]: 150; Tsujimura, 2007 [1996]: 17; Magnuson, 2008: 8; Vance, 2008: 56-57).

\subsection{Rasgos pertinentes}

Una vez examinadas las articulaciones vocálicas, pasemos a su descripción fonológica. Para empezar, partiremos de una afirmación ampliamente aceptada por la bibliografía consultada: el sistema vocálico del japonés se caracteriza por su simplicidad, ya que se compone de cinco unidades: /i/, /e/, /a/, /o/ y /u/ (Trubetzkoy, 1973 [1939]; Smith, 1980; Vance, 1987 y 2008; Rodríguez-Izquierdo, 1991; Hara, 1994; Itô y Mester, 1995; Akamatsu, 1997 y 2000; Hasegawa, 1999; Teshigawara, 2002; Hirayama, 2003; Ohata, 2004; Grenon, 2005; Saito, 2005; Ito, Kang y Kenstowicz, 2006; Kaneko, 2006; Tsujimura, 2007 [1996]; Magnuson, 2008; Crawford, 2009; Frellesvig, 2010; Labrune, 2012). El valor distintivo de estos cinco fonemas vocálicos queda demostrado con las siguientes oposiciones de palabras, en que cada una de las cinco vocales es suficiente, conmutándolas, para cambiar el significado de la palabra: 涇 /a/ 'mudo', 胃 /i/ 'estómago', 宇 /u/ 'contable para edificios', 絵 /e/ 'dibujo', お /o/ 'Oh'.

Para la determinación fonológica de las unidades vocálicas en japonés estándar se consideran dos aspectos fundamentales: el grado de abertura del tracto bucal, que da lugar a los valores [cerrado], [medio] y [abierto], y la configuración que adopta la lengua en la cavidad bucal, que origina los grados [anterior] y [posterior] (Smith, 1980; Akamatsu, 2000: 69-70; Hirayama, 2003: 128-131; Ohata, 2004: 4; Kaneko, 2006: 50; Tsujimura, 2007 [1996]: 17; Vance, 2008: 54; Labrune, 2012: 26). Otro de los aspectos con pertinencia fonológica en japonés es la duración vocálica, que distingue entre vocales breves y vocales largas (Smith, 1980; Rodríguez-Izquierdo, 1991: 122; Akamatsu, 1997: 45; Teshigawara, 2002: 50; Hirayama, 2003: 1; Grenon, 2005: 4; Ito, Kang y Kenstowicz, 2006: 73; Tsujimura, 2007 [1996]: 18; Vance, 2008: 56; Labrune, 2012: 25 y 39-40). Existen, asimismo, dos aspectos articulatorios sin per- 
tinencia funcional: el ensordecimiento vocálico y la deslabialización en las realizaciones de /u/ (Vance, 1987 y 2008; Hara, 1994; Akamatsu, 1997 y 2000; Hasegawa, 1999; Teshigawara, 2002; Hirayama, 2003; Maekawa, 2003; Kondo, 2005; Tsujimura, 2007 [1996]; Magnuson, 2008; Frellesvig, 2010; Labrune, 2012). Estas características fonéticas, lejos de establecer diferencias semánticas entre distintas lexías, lo único que provocan es la distinción diatópica del hablante (Akamatsu, 2000: 68 y 71).

En lo que respecta a la nasalidad de los sonidos vocálicos del japonés, diremos que esta es una mera característica fonética, sin repercusiones fonológicas. En japonés, al igual que en español, la acción del velo del paladar modifica los sonidos en determinados contornos, pero no presenta rendimiento funcional (Alarcos, 1986 [1965]: 66-68 y 145-150; Martínez Celdrán, 1989: 78-79; Quilis, 2006 [1993]: 145, 148-150 y 168-170; D’Introno, Del Teso y Weston, 1995: 144; Hidalgo y Quilis, 2004: 122-123, 2012: 159 y 162; Hualde, 2005: 123; Iribarren, 2005: 143-147; Gil, 2007: 428, 431 y 439; Martínez Celdrán y Fernández Planas, 2007: 171; RAE, 2011: 100-104). Lamentablemente, en ninguna de las obras examinadas para la descripción del sistema fonológico del japonés estándar se ofrece una descripción pormenorizada de tales contornos en japonés; simplemente, Akamatsu (1997: 57) se limita a aportar lo siguiente: "nasalization occurs as an automatic consequence of the contiguity of nasal consonants". Añade el ejemplo: 港 [m'ĩnãto] 'puerto'. En español pasan a realizarse como alófonos oronasalizados de los fonemas vocálicos orales, en caso de que la distribución contextual de los sonidos japoneses coincida con la española. De cualquier modo, no describiremos los contextos en que las vocales japonesas quedan impregnadas de nasalización, puesto que no es relevante para nuestro artículo, ya que la falta de funcionalidad de las unidades oronasalizadas en español queda patente en la ausencia de referentes gráficos en el sistema de escritura de la lengua española (Hualde, 2005: 7; RAE, 2010: 73).

\subsection{Transcripción de los sonidos vocálicos del japonés}

En cuanto al español, las fuentes consultadas (Canellada y Kuhlmann, 1987: 27; Martínez Celdrán, 1989: 78-79 y 2000: 143-144; D’Introno, Del Teso y Weston, 1995: 144; Hidalgo y Quilis, 2004: 122-123, 2012: 139-152, 159 y 162; Hualde, 2005: 120-124; Iribarren, 2005: 143-147; Quilis, 2006 [1993]: 144-148, 168-170; Gil, 2007: 431 y 439; Martínez Celdrán y Fernández Planas, 2007: 171; RAE, 2011: 76-88), siguen, en mayor o menor medida, la descripción aportada por Alarcos (1986 [1965]: 145-146) sobre los rasgos pertinentes para definir los fonemas vocálicos:

El español utiliza fonológicamente dos de las propiedades articulatorias y acústicas que sirven para la distinción de los fonemas vocálicos entre sí: a), el grado de abertura, que condiciona la mayor o menor frecuencia del llamado primer formante de la vocal, y b), la configuración de la cavidad bucal según la posición de la lengua y los labios, reflejada en la mayor o menor frecuencia del segundo formante de la vocal (timbre). 
De este modo, presentamos el siguiente cuadro descriptivo en el que comparamos ambos sistemas vocálicos. Los elementos fonológicos, esto es, con función distintiva, están sombreados con una tonalidad más oscura que el resto de elementos fonéticos. Mediante los símbolos $\rightarrow$ y X pretendemos mostrar la correlación con la lengua de destino: $\rightarrow$ indica la existencia de ese aspecto fonológico en la lengua de destino y X, la inexistencia o escasa repercusión.

\section{TABLA 1}

Comparación de los rasgos distintivos entre el sistema vocálico japonés y el español (I)

\begin{tabular}{ll}
\hline \multicolumn{1}{c}{$\begin{array}{l}\text { Grado de abertura: } \\
\text { [cerrado], [medio], [abierto] }\end{array}$} \\
\cline { 2 - 2 } VOCALES & $\begin{array}{l}\text { Configuración de la lengua: } \\
\text { [anterior], [posterior] }\end{array}$ \\
\cline { 2 - 2 } JAPONESAS & Longitud vocálica: \\
\cline { 2 - 2 } [larga], [breve]
\end{tabular}

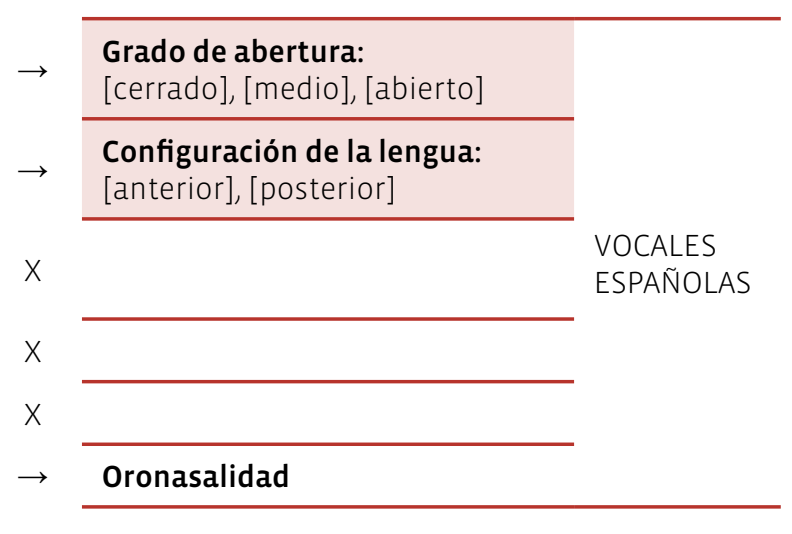

En la siguiente tabla establecemos una correlación entre las vocales breves del japonés y las unidades españolas, puesto que la longitud vocálica no es pertinente en español (Alarcos, 1986 [1965]: 145-146; Hualde, 2005: 124; Iribarren, 2005: 138; Gil, 2007: 441-443; RAE, 2011: 84, 112-113):

\section{TABLA 2}

Comparación de los rasgos distintivos entre el sistema vocálico japonés y el español (II)

\begin{tabular}{|c|c|c|c|c|c|}
\hline FONEMA & [ \pm CERRADO] & [ \pm MEDIO] & $\begin{array}{l}\text { [ } \pm \text { ABIERTO]/ } \\
\text { [+CENTRAL] }\end{array}$ & [ \pm ANTERIOR] & [ЕPOSTERIOR] \\
\hline /a/japonés & & & [+] (/i e o u/) & & \\
\hline /a/ español & & & [+] (/i e o o u/) & & \\
\hline /e/ japonés & & {$[+](/ \mathrm{i} /)$} & & {$[+](/ 0 /)$} & \\
\hline /e/ español & & {$[+](/ \mathrm{i} /)$} & & {$[+](/ 0 /)$} & \\
\hline /i/japonés & {$[+](/ e /)$} & & & {$[+](/ \mathrm{u} /)$} & \\
\hline /i/ español & {$[+](/ \mathrm{e} /)$} & & & {$[+](/ \mathrm{u} /)$} & \\
\hline /o/ japonés & & {$[+](/ \mathrm{u} /)$} & & & {$[+](/ e /)$} \\
\hline /o/ español & & {$[+](/ \mathrm{u} /)$} & & & {$[+](/ \mathrm{e} /)$} \\
\hline /u/ japonés & {$[+](/ 0 /)$} & & & & {$[+](/ e /)$} \\
\hline /u/ español & {$[+](/ 0 /)$} & & & & {$[+](/ e /)$} \\
\hline
\end{tabular}


Por tanto, si atendemos a la representación gráfica de los fonemas vocálicos en español de la RAE (2010: 72-85), deberíamos transcribir las vocales breves del japonés como sigue:

\section{TABLA 3}

Adaptación gráfica en español de las unidades vocálicas japonesas

\begin{tabular}{c|c}
$\begin{array}{c}\text { FONEMA } \\
\text { JAPONÉS }\end{array}$ & $\begin{array}{c}\text { TRANSCRIPCIÓN } \\
\text { GRÁFICA EN ESPAÑOL }\end{array}$ \\
\hline$/ \mathrm{a} /$ & $\mathrm{A}, \mathrm{a}$ \\
\hline$/ \mathrm{e} /$ & $\mathrm{E}, \mathrm{e}$ \\
\hline$/ \mathrm{i} /$ & $\mathrm{l}, \mathrm{i}$ \\
\hline$/ \mathrm{o} /$ & $\mathrm{O}, \mathrm{o}$ \\
\hline$/ \mathrm{u} /$ & $\mathrm{u}, \mathrm{u}$ \\
\hline
\end{tabular}

¿Qué ocurre con la transcripción ortográfica de las vocales largas? Teniendo en cuenta, por un lado, nuestro propósito de realizar un método de transcripción laxo y sencillo que solo tenga en cuenta los rasgos pertinentes de la lengua de destino - el español-, y, por otro, la unidireccionalidad de nuestro modelo (del japonés al español), no creemos necesario emplear signos diacríticos para indicar la longitud vocálica de los japonesismos. Además, creemos prácticamente imposible que un par de voces japonesas opuestas entre sí por la longitud vocálica se incluyan como préstamos léxicos en español; y de ser así, un hablante de español las reproduciría con vocal corta, puesto que la longitud vocálica no es pertinente en nuestra lengua ${ }^{10}$. Si alguna vez este caso ocurriera, bastaría con la aplicación del contexto y de otros elementos sintagmáticos, como los artículos y el género de los adjetivos adyacentes, para establecer diferencias entre una y otra lexía.

Hasta aquí, la transcripción vocálica desprende sencillez, pero el análisis se complica al profundizar en el estudio de /i/ y /u/, sobre todo en español, sombreadas en la tabla anterior. A priori, si creemos en la simplicidad de transcripción que caracteriza a las vocales /a/, /e/ y /o/ en español — pues no existen inconvenientes en su representación escrita, ya que siempre

10 En español no se produce un cambio de significado, ni se dificulta el entendimiento, si pronunciamos alcol en lugar de alcohol, ya que, según la RAE (2010: 164-172), es común la reducción de secuencias de dos vocales iguales. Al respecto, resultan esclarecedoras las declaraciones de Gil (2007: 449): "En España y en América se tiende a reducir cualquier grupo de sonidos vocálicos a una sola sílaba, o bien eliminando alguno de ellos — tanto si son iguales [...] como si son distintos [...] - o bien formando un diptongo a partir de un hiato original”. Esto responde a la tendencia antihiática de la que habla Quilis (2006 [1993]: 189-191), entre otros. 
se utilizan los grafemas a, e, o, respectivamente (RAE, 2010: 74) ${ }^{11}$ - es de esperar que /i/ y /u/ se transcriban con los grafemas i y u. No obstante, la relación sonido-grafía no es inequívoca o unitaria para la representación de los fonemas /i/ y /u/, puesto que en español se usan diferentes grafías para su transcripción ortográfica ${ }^{12}$.

Según la RAE (2010: 74 y 81), para representar el fonema/i/, debemos emplear "siempre la letra $i$, salvo en aquellos casos en que las normas prescriben o admiten explícitamente el uso de y", y para /u/ proyecta una idea similar: "La u es la letra genuinamente española para representar el fonema vocálico /u/, y puede hacerlo en cualquier posición”. Así, transcribiremos siempre con i y u todos los fonemas /i/ y /u/ que aparezcan en cualquier japonesismo, con independencia de su posición en la palabra; si bien ¿deberán seguir los japonesismos las excepciones expuestas en la RAE (2010: 74-87), que presentamos a continuación?:

\section{TABLA 4}

Excepciones para el fonema español/i/

(1) "El fonema /i/ situado a principio de palabra o de sílaba seguido de otra vocal con la que forma diptongo tiende a convertirse, en la pronunciación espontánea, en el fonema consonántico /y/, $[. .$.$] , algunas palabras que comienzan o contienen la secuencia h i$ - o $i$-seguida de vocal presentan variantes escritas con $y$-, letra a la que corresponde en el sistema ortográfico español la representación de dicho fonema consonántico" (RAE, 2010: 75).

(2) "Cuando se trata de la conjunción copulativa y" (RAE, 2010: 76).

(3) "Cuando, siendo este fonema átono o inacentuado, va en posición final de palabra precedido de una o dos vocales con las que forma un diptongo o un triptongo [...]. En cambio, si el fonema /i/ final precedido de vocal es tónico, forma hiato o diptongo con la vocal precedente, se representa con la letra $i[. .$.$] . La regla que prescribe el uso de -y cuando el fonema /i/ final no es tónico y va$ precedido de otra vocal presenta algunas excepciones, todas ellas en palabras procedentes de otras lenguas que, aun cumpliendo dicha condición, se escriben con -i [...]. En ciertos términos procedentes asimismo de otras lenguas, se admiten ambas grafías, con -y y con -i, siendo la preferida en el uso la citada en primer lugar, que es, por ello, la forma recomendada”" (RAE, 2010: 78) ${ }^{13}$.

(4) En casos excepcionales: en formas verbales, en algunos topónimos y antropónimos autóctonos o foráneos y en sus derivados, y, por último, en siglas y acrónimos (RAE, 2010: 79-80).

11 Salvo en los casos de $h$ inicial, $h$ intercalada o $h$ final; el grafema $h$, que no representa ningún fonema en español actual, responde a criterios etimológicos, expresivos - interjección-o extranjerismos; estos tres casos no afectan a los japonesismos adoptados en español, por lo que no lo tendremos en cuenta.

12 La variedad gráfica que encontramos en el español actual podría deberse a varios factores: por un lado, la etimología de las voces. Por otro lado, la indeterminación fonética de estas unidades: ¿fonemas o alófonos?, ¿consonantes, vocales u otro tipo de unidad? Preguntas que constituyen la base de la disputa actual entre fonólogos y fonetistas.

13 Al respecto, leemos en la recomendación c) de la RAE (2010: 621): "Si la transcripción del original presenta una i precedida de vocal en posición final de palabra, suele mantenerse: [...], bonsái, samurái; [...] aunque resultaría más apropiado transformarla en -y, que es la grafía normal en español para representar el fonema/i/ en esta posición”. 


\section{TABLA 5}

Excepciones para el fonema español/u/

(1) "Cuando el fonema /u/ aparece en las secuencias fónicas /gue/, /gui/, la letra u que lo representa debe escribirse con diéresis" (RAE, 2010: 81).

(2) "En español, con muy pocas excepciones, se escribe $h$ delante de /u/ + vocal, tanto a principio de palabra como en posición interior a comienzo de sílaba” (RAE, 2010: 82).

(3) "En las palabras que contienen los diptongos /ua, / /ue/, /ui/a principio de palabra o en posición interior a comienzo de sílaba [...] se suele articular ante el diptongo un refuerzo consonántico cercano al fonema /g/ [...]. Este hecho ha alcanzado a veces reflejo en la escritura [...]. Cabe decir que, en general, las formas con h suelen ser las preferidas en la lengua culta" (RAE, 2010: 82-83).

(4) "La letra w representa el fonema vocálico /u/ — o la secuencia con refuerzo consonántico /gu/ cuando dicho fonema forma diptongo con la vocal siguiente a comienzo de palabra o de sílaba- en la mayoría de las palabras de origen inglés en las que se ha conservado esta letra en su paso al español, así como en ciertos términos transcritos al alfabeto latino procedentes de lenguas orientales, semíticas o indígenas, introducidos muchos también a través del inglés [...]. Puesto que la letra $u$ es el grafema genuinamente español para representar el fonema/u/, algunas voces foráneas con $w$ en su grafía originaria se han adaptado al español trocando la $w$ en $u$ [...]. En algún caso se dan por válidas ambas grafías” (RAE, 2010: 85-86).

Observamos que la excepción (1) del fonema /i/ en español está relacionada con un problema actual que concierne a la delimitación fonológica de los elementos semiconsonánticos, [j] y [w], en castellano. Como así se describe en el manual de fonética y fonología de la RAE:

Durante los últimos años se han producido intensos debates acerca de la naturaleza fonológica de esta clase de segmentos. Para algunos autores, las vocales marginales son segmentos independientes de las vocales, se deberían transcribir con los símbolos /j/ y /w/, y figurarían como dos unidades más en el inventario de segmentos contrastivos del español14. Para otros, esas vocales son variantes contextuales de /i/ y /u/, y carecen de valor distintivo ${ }^{15}$.

Los defensores de la primera postura, que otorga estatuto fonológico independiente a las vocales marginales, aducen argumentos de carácter fonético para sostenerla. Según estos autores, existen suficientes diferencias acústicas, de carácter temporal y relativas a la frecuencia, entre las vocales silábicas y las marginales para postular la existencia de dos segmentos contrastivos de este último tipo en el sistema del español. Los defensores de la segunda postura sostienen que la naturaleza de las vocales marginales está determinada por la posición silábica, por lo que la aparición de un sonido de estas características es contextualmente predecible; además, no existen en español pares mínimos que se diferencien únicamente por la presencia de una vocal satélite o de la vocal silábica correspondiente. Así, estos autores consideran que una vocal marginal o satélite es una

14 Principalmente, se refiere la Academia a la vertiente iniciada por Martínez Celdrán (1989: 79-84; 2007: 161-170) y sus seguidores, Hidalgo y Quilis (2004: 129, 136-144; 2012: 162-171), entre otros.

15 Un clarísimo referente de este movimiento es Alarcos (1986 [1965]: 152-160) y todos sus defensores: Canellada y KuhImann (1987: 25), Quilis (2006 [1993]: 182-183) e Iribarren (2005: 155-162), entre otros. 
realización fonética determinada por el proceso de silabación.

Como se deduce de los apartados anteriores, el estatuto fonológico de las vocales marginales es un problema de difícil solución (RAE, 2011: 342-343).

En español, pues, existe una indeterminación en cuanto al estatus fonológico de estas unidades, que varía según la escuela o el autor. Fiel reflejo de tal desacuerdo, que se había venido gestando a lo largo de la historia del alfabeto español — por lo que es muy anterior a los análisis fonológicos-, es la representación gráfica de estas unidades.

Por un lado, para/i/ inicial se fluctúa entre $i$-, hi- o y-, si bien las formas preferidas, por ser el uso mayoritario en el conjunto hispánico, son hi- o i- (RAE, 2010: 75). En japonés, al tratarse del elemento vocálico /i/, siempre lo transcribiremos mediante la grafía i-, pues hi-, contiene un grafema, $h$, antietimológico. Otra excepción especial del fonema /i/, la número (3), prescribe que la forma preferida para /i/ átona en posición final de palabra y precedida de una o dos vocales con las que forma un diptongo o un triptongo sea $-y$. Siguiendo los consejos anteriores, /i/ será transcrito como $i$ en todos los contextos, salvo en el recién explicado, esto es, como elemento átono final de un diptongo o triptongo en final de palabra. En plural, no

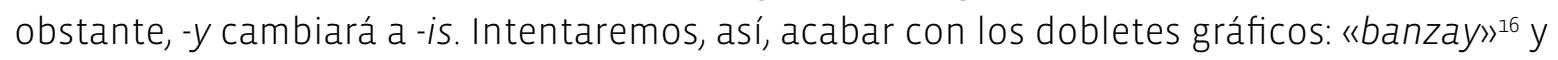
no «banzái», «bonsay»y no «bonsái», «jaicay» y no «jaicái», «samuray» pero no «samurái».

La distribución similar que comparte con /i/ ha provocado que para la transcripción de /u/ existan ciertas particularidades gráficas. A pesar de dichas excepciones expresadas en (2), (3) y (4), siempre transcribiremos /u/ procedente del japonés con la grafía u por los siguientes motivos: por un lado, damos unidad — al igual que en el caso de /i/ y el resto de unidades vocálicas-, por lo que el sistema de transcripción para las vocales queda simplificado y libre de particularidades, haciéndolo accesible no solo a los hablantes nativos, sino también a los extranjeros que deseen aprender nuestro idioma. Además, el uso de ho g delante de /u/ + vocal es antietimológico para las voces procedentes del japonés. En definitiva, transcribiremos siempre mediante $u$, grafema que mejor refleja el estatus vocálico del étimo japonés.

\section{Unidades semivocálicas del japonés}

El japonés actual cuenta con dos unidades semivocálicas, /j/ y /u/. En lo que respecta a la articulación de [j], con las mismas cualidades fónicas que las de [i], se caracteriza porque el

16 Como acordamos en nuestra tesis, por convención gráfica, transcribiremos los japonesismos que han pasado por nuestro filtro transcriptivo entre comillas, «», indicando mediante la cursiva si todavía son meros extranjerismos. Para conocer el grado de adaptación de los japonesismos, vid. Fernández Mata (2017). 
órgano móvil no permanece en la posición para producir [i] durante mucho tiempo, sino que rápidamente cambia de estado para adoptar la cualidad de la vocal siguiente (Akamatsu, 1997: 43, 2000: 96; Vance, 2008: 89). En relación a este último aspecto, esta unidad solo aparece ante las vocales /a/, /o/ y /u/: 矢 [ja] ‘flecha', 夜 [jolu] 'noche', 雪 [juk'i] 'nieve' (Akamatsu, 1997: 43, 2000: 96; Ito, Kang y Kenstowicz, 2006: 18; Vance, 2008: 90; Labrune, 2012: 88). Como sostienen Akamatsu (1997: 43), Ito, Kang y Kenstowicz (2006: 18) y Labrune (2012: 88), la combinación [ji] es imposible en cualquier estrato. En cuanto a [je], Akamatsu (1997: 43), Ito, Kang y Kenstowicz (2006: 18) y Labrune (2012: 88) describen que se encuentra ausente, salvo en préstamos muy recientes, en algunos dialectos - en particular, los de la isla de Quiusiu-, por lo que no forma parte funcional del japonés estándar. En efecto, como demuestra Frellesvig (2010: 387), la reducción de [je] o [je] a [e] fue contemporánea o posterior al periodo Meyi (1868-1912).

En cuanto a [u], se describe su articulación con los valores fónicos [-consonante, +sonante], [+sonoro], [+posterior], es decir, comparte prácticamente las cualidades del sonido vocálico [w] (Akamatsu, 1997: 44; Vance, 2008: 89)17. Akamatsu (2000: 97-98, 137-139), Vance (2008: 89-92) y Labrune (2012: 90-92) examinan la distribución de [u] y llegan a la conclusión de que el segmento [u] solo se da ante /a/ en [ua] (Akamatsu, 1997: 44, 2000: 97-98; Vance, 2008: 90; Labrune, 2012: 90), pues las secuencias [щi], [ще] y [що], que existieron en estadios primitivos de la lengua ${ }^{18}$, han desaparecido en japonés moderno ${ }^{19}$. Estiman, asimismo, que nunca existió la secuencia [um] (Akamatsu, 1997: 44, 2000: 97; Vance, 2008: 90; Labrune, 2012: 90)20. En la última mitad del siglo XX y durante las primeras décadas del siglo XXI, debido a la gran oleada de préstamos lingüísticos adoptados por el japonés - la gran mayoría procedentes de la lengua inglesa-, las secuencias [ui], [ще] y [uo] han aumentado su aparición dentro del inventario fonético de la lengua japonesa, pero fluctúan con las combinaciones [ui], [ue] y [mo] (Akamatsu, 2000: 99-100; Vance, 2008: 91-92; Labrune, 2012: 90).

Existen, pues, varias pruebas que demuestran el estatus fonológico de /j/y/u/. En primer lugar, presentan un esquema silábico fijo y determinado, como el de las unidades no vocálicas o consonánticas. Aunque a diferencia de estas, no presentan correlatos palatalizados, característica que comparten con las unidades vocálicas. Asimismo, existe rendimiento funcional

17 Discrepa levemente Labrune (2012: 90): "its phonetic realization is between that of the symbols [u] and $[\mathrm{w}]$ of the IPA".

18 Según Frellesvig (2010: 310), la total desaparición del elemento velar ante /e i o/ se había completado en torno a 1300.

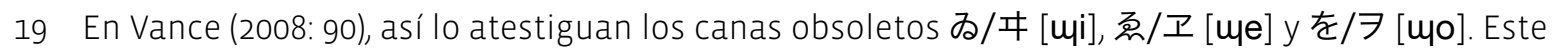
último solo se usa para la partícula de objeto directo /o/. Sobre este último aspecto, comenta Labrune (2012: 90): "The object particle is sometimes romanized as wo, but this is a purely orthographic convention, that does not reflect the presence of the glide / $w /$ in the actual realization of the particle".

20 Vance (2008: 90) añade que nunca existieron canas para esta combinación. 
de pares léxicos en oposición (como demuestran Akamatsu, 2000; Vance, 2008 y Labrune, 2012). Desde el punto de vista de su representación silábico-gráfica, aparecen siempre combinados con las unidades vocálicas a las que preceden, al igual que el resto de sonidos no vocálicos. Por tanto, constituyen una categoría diferente a la compuesta por las unidades vocálicas y las no vocálicas o consonánticas, aunque comparten con las primeras ciertas cualidades fónicas y con las segundas, características distributivas.

\subsection{Transcripción de los sonidos semivocálicos del japonés}

Las unidades semivocálicas /j/ y / $\mathbf{m / ~ c o n s t i t u y e n ~ u n ~ s e r i o ~ i n c o n v e n i e n t e ~ a ~ l a ~ h o r a ~ d e ~ s u ~ t r a n s - ~}$ cripción gráfica en español. ¿Debemos utilizar los grafemas representativos de las unidades vocálicas, i y $u$, o las letras que transcriben unidades consonánticas como y o g?

Dado que, por un lado, la RAE (2010) da preferencia a los grafemas vocálicos i y u o a las combinaciones hi y hu para la representación de los sonidos vocálicos, [i]-[u], y de los sonidos semiconsonánticos, [j]-[w], en lengua española, y que, por otro, los sonidos semiconsonánticos de la lengua española son muy próximos a las realizaciones de las unidades semivocálicas japonesas, /j/ y / w/, creemos que la solución más práctica es adoptar la convención gráfica de la RAE (2010) para transcribir las semivocales japonesas. Sin embargo, no utilizaremos ni hi ni hu, por su grafema antietimológico $h$, que no procede de la lengua japonesa. Cierto es que en español el sonido semiconsonántico [w] presenta cualidades fónicas cercanas a las consonantes y que esto se traduce en español con un refuerzo de la articulación, bien con gu- o bien con w-; no obstante, la RAE (2010: 83 y 86) prefiere el uso de hu-y se mantiene ambigua con las voces transcritas con $w$ - (por influencia del inglés), ya que da por válidas las grafías $u$ - o $w$-. En nuestro caso no existe este problema, ya que

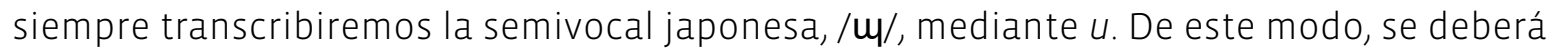
escribir «iaquitori» y no «yakitori» o «jakitori», «uasabi» y no «wasabi» o «guasabi», «ien»y no «yen»o «jen», «iucata»y no «yukata»o «jukata».

\section{Unidades consonánticas del japonés}

Pese a que nos basamos principalmente en las obras de Akamatsu (1997 y 2000) y de Ladefoged y Maddieson (1996), hemos considerado las aportaciones de los siguientes autores para el estudio del sistema consonántico del japonés moderno: Smith, 1980; Rodríguez-Izquierdo, 1991; Hara, 1994; Itô y Mester, 1995; Hasegawa, 1999; Teshigawara, 2002; Hirayama, 2003; Ohata, 2004; Grenon, 2005; Kondo, 2005; Saito, 2005; Ito, Kang y Kenstowicz, 2006; Kaneko, 2006; Kim, 2007; Tsujimura, 2007 [1996]; Levitt y Katz, 2008; Magnuson, 2008, 2009, 2011; Crawford, 2009; Kubozono, Itô y Mester, 2009; Frellesvig, 2010; Nogita, 2010; Labrune, 2012. 
Tras analizar el material bibliográfico, concluimos en que el japonés actual cuenta con un inventario de 23 unidades consonánticas, divididas entre unidades palatalizadas y unidades no palatalizadas (Akamatsu, 2000: 84-85, 105-127, 129, 183; Tsujimura, 2007 [1996]: 15; Vance, 2008: 92-93, 225-232; Labrune, 2012: 88-90, 144). Según Tsujimura (2007 [1996]: 15), Akamatsu (1997: 20), Alonso-Cortés (2002: 143) y Vance (2008: 230), la palatalización implica una articulación secundaria que tiene lugar cuando la lengua adopta la forma para producir [i], mientras articula un elemento de carácter no vocálico; o, lo que es lo mismo, se eleva la zona delantera lingual hacia el paladar duro, que no llega a tocar, mientras se articulan algunas unidades fónicas ${ }^{21}$. Asimismo, la oposición entre las unidades palatalizadas y sus correlatos no palatalizados se produce siempre ante las vocales /a/, /o/ y /u/ (Akamatsu, 2000: 88; Vance, 2008: 93).

Existen, según el lugar de articulación, cuatro órdenes consonánticos en japonés ${ }^{22}$. El orden labial, en el que las consonantes se caracterizan por la acción de los órganos externos labiales (Ladefoged y Maddieson, 1996: 44; Akamatsu, 2000: 90-91). El orden coronal, donde las consonantes se articulan con el ápice, predorso o dorso de la lengua y la zona que comprende desde los incisivos superiores hasta la zona alveopalatal (Ladefoged y Maddieson, 1996: 44; Akamatsu, 1997: 78-82, 118-120; 2000: 91-92). El orden dorsal, en el que las consonantes son pronunciadas mediante la acción del dorso o posdorso lingual contra el paladar duro o el velo del paladar (Ladefoged y Maddieson, 1996: 44; Akamatsu, 2000: 91). Por último, el orden laríngeo, lugar donde se producen los elementos glóticos (Ladefoged y Maddieson, 1996: 44; Akamatsu, 2000: 92).

Con respecto al modo de articulación de las consonantes japonesas, podemos establecer cuatro tipologías de segmentos consonánticos. De un lado, obstruyentes u oclusivos: el flujo de aire encuentra un impedimento en forma de cierre total. Por otro, constrictivos o fricativos: el flujo de aire encuentra un obstáculo en forma de fricción. También, africados: una combinación de los dos anteriores, esto es, se produce consecutivamente una oclusión y una constricción (por ejemplo, el caso de [ts]). Durante la producción de los sonidos aproximantes el flujo de aire atraviesa el tracto bucal sin producir una fricción similar a la de los sonidos fricativos, pero con un grado de abertura inferior al de las realizaciones de los sonidos vocálicos. Siguiendo la propuesta de Martínez Celdrán y Fernández Planas (2007: 123), no clasificaremos las nasales con las oclusivas, "puesto que constituyen un modo particular diferente tanto de las oclusivas como de las continuas”. Estos autores siguen las coordenadas expuestas por Ladefoged y Maddieson (1996: 103): "Nasals have an articulatory similarity to stops by virtue of

21 Ladefoged y Maddieson (1996: 363): "Palatalization is the superimposition of a raising of the front of the tongue toward a position similar to that for i on a primary gesture".

22 Por su extensión, nos resulta imposible ofrecer una descripción detallada de las unidades consonánticas del japonés actual desde un punto de vista articulatorio, si bien se podrán observar sus rasgos articulatorios en el desarrollo de nuestro sistema de transcripción al español. 
their oral closure, but in other respects they are similar to approximants. This is because there is an uninterrupted outward flow of air that does not pass through a constriction sufficiently narrow to produce local turbulence. There are no fricative nasals".

Martínez Celdrán y Fernández Planas (2007: 123) creen que son concomitantes las unidades oclusivas en la cavidad oral y las continuas en la cavidad nasal, porque solo pueden ser "verdaderas consonantes nasales aquellas que poseen un cierre bucal y una abertura rinofaríngea”. Por tanto, el modo de articulación del japonés incluirá la oclusión, la fricción, la africación ${ }^{23}$, la aproximación y la nasalidad. Casi todos los sonidos consonánticos se caracterizan por no poner en funcionamiento la cavidad complementaria nasal, por lo que poseen el rasgo [-nasal]. Existen en japonés ocho sonidos nasales: [m], [m’], [n], [n’], [n], [n'], [n] y [ñ] (Akamatsu, 1997: 134 y 138).

Por último, según la acción de las cuerdas vocales, los segmentos consonánticos del japonés se pueden caracterizar por la presencia o ausencia de sonoridad, [ะsonoro].

A continuación, desarrollamos una tabla en la que ilustramos - mediante el modo, la zona de articulación y la acción de las cuerdas vocales²4_ cuáles son las unidades consonánticas del inventario fonológico del japonés actual:

\section{TABLA 6}

Descripción esquemática de las unidades consonánticas japonesas según la zona de articulación, el modo de articulación y la acción de las cuerdas vocales

\begin{tabular}{|c|c|c|c|c|c|}
\hline & & & ZONA DE AR & CULACIÓN & \\
\hline & & [+labial] & [+coronal] & [+dorsal] & [+laríngeo] \\
\hline & OCLUSIVAS & /p/-/p'/ : /b/-/b'/ & $/$ th/-/t'/ $1 / d /$ & $\begin{array}{l:l}/ k /-/ k^{\prime} / & / g /-/ g^{\prime} /\end{array}$ & i \\
\hline 철홈 & FRICATIVAS & 1 & $|s /-| s^{\prime} /|| z|-| z^{\prime} \mid$ & 1 & /h/-/h'/ I \\
\hline 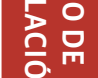 & APROXIMANTES & 1 & | / / / & 1 & 1 \\
\hline & NASALES & I /m/-/m'/ & I /n/-/n'/ & I & i \\
\hline & & $\begin{array}{l:l}{[-]} & {[+]}\end{array}$ & {$\left[\begin{array}{l:l}-] & {[+]}\end{array}\right.$} & {$[-] \quad: \quad[+]$} & {$[-] \quad: \quad[+]$} \\
\hline
\end{tabular}

23 Aunque como convinimos en nuestra tesis inédita sobre Los japonesismos de la lengua española: Historia y transcripción, la africación no posee valor fonológico.

24 El elemento de la izquierda posee el rasgo [-sonoro], mientras que el de la derecha es [+sonoro]. 


\subsection{Nuestro método de transcripción}

Para la transcripción ortográfica de las unidades no vocálicas del japonés realizaremos una subdivisión. En primer lugar, trataremos las unidades sin palatalización. Dentro de esta categoría, dividiremos los fonemas siguiendo los órdenes consonánticos que acabamos de apuntar, esto es, consonantes labiales, consonantes coronales, consonantes dorsales y consonante faríngea. En último lugar, estudiaremos algunos fenómenos especiales dentro de las unidades no vocálicas y su representación en nuestro sistema gráfico.

\subsubsection{Unidades consonánticas sin palatalización}

A continuación, trataremos de relacionar cada una de las unidades no palatalizadas japonesas con los elementos del sistema fonológico del español más similares, para lo que tendremos presente las cualidades fonéticas de todos estos segmentos. En este sentido, seguiremos un amplio abanico de bibliografía sobre fonética y fonología del español (Alarcos, 1986 [1965]: 161-179; Canellada y KuhImann, 1987: 19-24, 36-39; Martínez Celdrán, 1989: 84-104; 1996: 5-16; 2000: 144-153; D'Introno, Del Teso y Weston, 1995: 259-313; Hidalgo y Quilis, 2004: 145-201; 2012: 173-237; Navarro Tomás, 2004 [1918]: 77-145; Hualde, 2005: 138-189; Iribarren, 2005: 165-175, 193324; Quilis, 2006 [1993]: 194-359; Gil, 2007: 479-519; Martínez Celdrán y Fernández Planas, 2007: 31-170; RAE, 2011: 115-263), pero siempre daremos preferencia a los estudios funcionalistas, ya que este ha sido nuestro método básico de descripción para el sistema fonológico del japonés.

\subsubsection{Los fonemas labiales}

En el orden de las labiales encontramos tres fonemas sin palatalización: /p/, /b/ y /m/, cuyas cualidades fónicas resumiremos en la próxima tabla comparativa:

\section{TABLA 7}

Comparación de los rasgos distintivos entre las unidades bilabiales japonesas y sus españolas más próximas

\begin{tabular}{|c|c|c|c|c|}
\hline FONEMA & [ESONORO] & {$[ \pm N A S A L]$} & [ \pm OCLUSIVO] & [ \pm LABIAL] \\
\hline /p/ japonés & {$[-]$} & {$[-]$} & {$[+]$} & {$[+]$} \\
\hline /p/ español & {$[-]$} & {$[-]$} & {$[+]$} & {$[+]$} \\
\hline /b/ japonés & {$[+]$} & {$[-]$} & {$[+]$} & {$[+]$} \\
\hline /b/ español & {$[+]$} & {$[-]$} & {$[ \pm]^{25}$} & {$[+]$} \\
\hline /m/japonés & {$[+]$} & {$[+]$} & {$[+]$} & {$[+]$} \\
\hline /m/ español & {$[+]$} & {$[+]$} & {$[+]$} & {$[+]$} \\
\hline
\end{tabular}


Según las recomendaciones de la RAE (2010: 87-164)26 para la representación gráfica de los fonemas consonánticos en español, deberíamos transcribir las labiales de los préstamos japoneses de la siguiente manera:

\section{TABLA 8}

Adaptación gráfica en español de las unidades bilabiales japonesas

\begin{tabular}{c|c}
$\begin{array}{c}\text { FONEMA } \\
\text { JAPONÉS }\end{array}$ & $\begin{array}{c}\text { TRANSCRIPCIÓN } \\
\text { GRÁFICA EN ESPAÑOL }\end{array}$ \\
\hline$/ \mathrm{p} /$ & $\mathrm{P}, \mathrm{p}$ \\
\hline $\mathrm{b} / \mathrm{B}, \mathrm{b}$ \\
\hline$/ \mathrm{m} /$ & $\mathrm{M}, \mathrm{m}$ \\
\hline
\end{tabular}

En cuanto a la transcripción del fonema /b/ en español — sombreado en la tabla-, en esta lengua /b/ puede ser representado mediante varias letras: $b, v$ y $w$, de las que $b$ y $v$ son herencia latina y $w$, inexistente en latín, tiene lugar únicamente en la representación de voces extranjeras (RAE, 2010: 91). Ya que es imposible "determinar la presencia de b o $v$ en representación del fonema /b/ sin conocer de antemano la grafía de cada palabra" (RAE, 2010: 94), nosotros siempre utilizaremos la grafía b para los préstamos de origen japonés que contengan dicho sonido, puesto que $v$ sirvió en tiempos pretéritos del alfabeto español para la representación de un sonido bilabial, fricativo ([ß]) o labiodental ([V]), fricativo, sonoro (Lapesa, 2005 [1981]: 195-196; Alarcos, 1986 [1965]: 258-259; RAE, 2010: 91-92). De esta forma, nuestro sistema de transcripción usará elementos propiamente españoles y ganará en sencillez. Así, encontramos japonesismos transcritos con b como: «biombo», «bonsay», «bonzo», «cabuqui», «iquebana»o «misubisi», entre otros.

\subsubsection{Los fonemas coronales}

El orden de las consonantes coronales del japonés cuenta con dos unidades no palatalizadas de oclusión oral (/t/, /d/), dos fonemas fricativos sin palatalización (/s/, /z/27), un segmento

25 En español puede ser oclusivo o aproximante, dependiendo del contexto.

26 De aquí en adelante, usaremos siempre las mismas páginas para determinar la transcripción de los demás sonidos consonánticos.

27 Se articula [z] o [cz] ante las unidades vocálicas /e/, /a/, /o/ y /u/: 税 [ze] o [cze] 'impuestos', 風 見 [kazam'i] o [kaczam'i] 'veleta', 画像 [gazo:] o [gaczo: 'imagen', 数 [kazu] o [kaczu] 'número' (Tsujimura, 2007 [1996]: 33-34; Akamatsu, 1997: 95-96; Vance, 2008: 88; Labrune, 2012: 64). Las realizaciones fricativas o africadas del fonema /z/ se dan tanto en inicial de palabra como en posición 
aproximante y otro oclusivo nasal no palatalizados (/d/28 y $/ \mathrm{n} /$ ). Asimismo, encontramos el caso especial de la unidad [ts], que no constituye unidad distintiva en el inventario fonológico del japonés, sino que es alófono de/t/ante/u/. Desde el punto de vista fónico, estas unidades comparten muchas cualidades con las consonantes españolas que se articulan de manera coronal.

\section{TABLA 9}

Comparación de los rasgos distintivos entre la coronal japonesa /t/ y su española más próxima

\begin{tabular}{c|c|c|c|c} 
FONEMA & {$[ \pm$ SONORO] } & {$[ \pm$ NASAL] } & {$[ \pm$ OCLUSIVO] } & {$[ \pm$ CORONAL] } \\
\hline /t/ japonés & {$[-]$} & {$[-]$} & {$[+]$} & {$[+]$} \\
\hline /t/ español & {$[-]$} & {$[-]$} & {$[+]$} & {$[+]$} \\
\hline
\end{tabular}

\section{TABLA 10}

Comparación de los rasgos distintivos entre la coronal japonesa/ts/ y su española más próxima

\begin{tabular}{c|c|c|c|cc} 
FONEMA & {$[ \pm$ SONORO $]$} & {$[ \pm$ NASAL $]$} & {$[ \pm$ AFRICADO $]$} & {$[ \pm$ FRICATIVO $]$} & {$[ \pm$ CORONAL] } \\
\hline [ts japonés & {$[-]$} & {$[-]$} & {$[+]$} & & {$[+]$} \\
\hline /s/español & {$[ \pm]^{29}$} & {$[-]$} & & {$[+]$} & {$[+]$} \\
\hline
\end{tabular}

\section{TABLA 11}

Comparación de los rasgos distintivos entre la coronal japonesa /d/ y su española más próxima

\begin{tabular}{c|c|c|c|c} 
FONEMA & {$[ \pm$ SONORO] } & {$[ \pm$ NASAL] } & {$[ \pm$ OCLUSIVO] } & {$[ \pm$ CORONAL] } \\
\hline$/$ d/ japonés & {$[+]$} & {$[-]$} & {$[+]$} & {$[+]$} \\
\hline /d/ español & {$[+]$} & {$[-]$} & {$[ \pm]^{30}$} & {$[+]$} \\
\hline
\end{tabular}

intervocálica, sin embargo, hay una mayor tendencia a pronunciar [cz] en posición inicial y [z] en situación intervocálica (Akamatsu, 1997: 95; Vance, 2008: 85; Labrune, 2012: 64). Según Tsujimura (2007 [1996]: 13), a pesar de que no hay claro contraste entre [dz] y [z], se puede observar la aparición consistente de uno u otro sonido dependiendo del contexto léxico, pues se prefiere uno para algunas palabras y otro para otras lexías.

28 En nuestra tesis empleamos [ 1$]$ — esta grafía fue propuesta por Okada (1999), aunque ya la encontramos en Ladefoged y Johnson (2011 [1975]: 179)-, que no aparece en el AFI —en el AFI aparece un símbolo muy parecido, $\lambda$, descrito como aproximante, alveolar, sonoro, que se caracteriza por su menor longitud en comparación con $\downarrow$-. Apréciese que se representa como la fusión de una $r$ invertida y una $l$, lo que nos pareció acertado desde el primer momento, dada su naturaleza fónica.

29 Posee realizaciones sordas y sonoras.

30 En español puede ser oclusivo o aproximante, dependiendo del contexto. 


\section{TABLA 12}

Comparación de los rasgos distintivos entre la coronal japonesa /s/ y su española más próxima

\begin{tabular}{c|ccccc} 
FONEMA & {$[ \pm$ SONORO $]$} & {$[ \pm$ NASAL] } & {$[ \pm$ FRICATIVO] } & {$[ \pm$ CORONAL] } \\
/S/ japonés & {$[-]$} & {$[-]$} & {$[+]$} & {$[+]$} \\
\hline /S/ español & {$[ \pm]$} & {$[-]$} & {$[+]$} & {$[+]$} \\
\hline
\end{tabular}

\section{TABLA 13}

Comparación de los rasgos distintivos entre la coronal japonesa /z/ y su española más próxima

\begin{tabular}{c|ccc|cc} 
FONEMA & {$[ \pm$ SONORO] } & {$[ \pm$ NASAL] } & {$[ \pm$ FRICATIVO] } & {$[ \pm$ CORONAL] } & {$[ \pm$ INTERDENTAL] } \\
/z/ japonés & {$[+]$} & {$[-]$} & {$[+]$} & {$[+]$} & \\
\hline$/ \theta /$ español & {$[ \pm]^{31}$} & {$[-]$} & {$[+]$} & & {$[+]$} \\
\hline
\end{tabular}

\section{TABLA 14}

Comparación de los rasgos distintivos entre la coronal japonesa / d/ y su española más próxima

\begin{tabular}{|c|c|c|c|c|c|}
\hline FONEMA & [ \pm SONORO] & [ \pm NASAL] & [士APROXIMANTE] & [ \pm PERCUSIVO] & [ \pm CORONAL] \\
\hline /d/ japonés & {$[+]$} & {$[-]$} & {$[+]$} & & {$[+]$} \\
\hline /ז/ español & {$[+]$} & {$[-]$} & & {$[+]$} & {$[+]$} \\
\hline
\end{tabular}

\section{TABLA 15}

Comparación de los rasgos distintivos entre la coronal japonesa /n/ y su española más próxima

\begin{tabular}{ccccc} 
FONEMA & {$[ \pm$ SONORO] } & {$[ \pm$ NASAL] } & {$[ \pm$ CORONAL] } \\
\hline$/ n /$ japonés & {$[+]$} & {$[+]$} & {$[+]$} \\
\hline$/ n /$ español & {$[+]$} & {$[+]$} & {$[+]$} \\
\hline
\end{tabular}

31 La realización japonesa es sonora y la española presenta también contextos donde se articula con sonoridad, por tanto, creemos que el equivalente fónico más similar a la unidad japonesa /z/ es / $\theta /$. Sabemos que las realizaciones fricativas [z] o africadas [cz] del fonema japonés /z/ se presentan tanto en inicial de palabra como en posición intervocálica, sin embargo, hay una mayor tendencia a pronunciar [cz] en posición inicial y [z] en situación intervocálica. Precisamente, es la realización africada la que, por presentar el obstáculo total entre el predorso lingual y parte de la cara interior de los incisivos superiores y la protuberancia alveolar próxima a estos, resulta articulatoriamente próxima a nuestra $[\theta]$. 
Los sonidos coronales se transcriben en español como sigue:

\section{TABLA 16}

Adaptación gráfica en español de las unidades coronales japonesas

FONEMA

JAPONÉS

\begin{tabular}{cc}
\hline$/ \mathrm{t} /$ & $\mathrm{T}, \mathrm{t}$ \\
\hline$/ \mathrm{d} /$ & $\mathrm{D}, \mathrm{d}$ \\
\hline$[\mathrm{ts}]$ & $\mathrm{TS}, \mathrm{ts}, \mathrm{S}, \mathrm{s}$ \\
\hline$/ \mathrm{s} /$ & $\mathrm{S}, \mathrm{s}$ \\
\hline$/ \theta /$ & $\mathrm{Z}, \mathrm{z}(+/ \mathrm{a} \circ \mathrm{u} /), \mathrm{C}, \mathrm{c}(+/ \mathrm{e} \mathrm{i} /)$ \\
\hline$/ \mathrm{d} /$ & $\mathrm{R}, \mathrm{r}$ \\
\hline$/ \mathrm{n} /$ & $\mathrm{N}, \mathrm{n}$ \\
\hline
\end{tabular}

No hallamos información en la RAE (2010) acerca de cómo transcribir la realización [ts]. Sin embargo, leemos en las advertencias de grafemas para el fonema /s/:

La secuencia sh es un dígrafo con el que se representa gráficamente el fonema prepalatal fricativo sordo /sh/, inexistente en el sistema fonológico del español actual. Puesto que ni el fonema /sh/ [...] ni el dígrafo sh que lo representan pertenecen al sistema fonológico ni gráfico del español, los préstamos que los contienen no pueden considerarse adaptados a nuestra lengua y deben, por ello, escribirse en cursiva [...].

Los extranjerismos de este tipo, cuando se han adaptado al español, lo han hecho asimilando el fonema /sh/ originario a alguno de los más próximos del sistema fonológico del español, normalmente/ch/ (gráficamente ch) o/s/ (gráficamente s) (RAE, 2010: 127).

Por tanto, si aplicamos esta recomendación de manera paralela a [ts], o bien transcribimos [ts] mediante el dígrafo ts, escribiendo la palabra en cursiva, o utilizamos el sonido más próximo en español, en este caso representado por el fonema/s/y su grafía s. De este modo, según el Diccionario de la Real Academia Española (RAE, 2014, vigesimotercera edición), en español actual conviven tsunami - extrañamente sin cursiva - con sunami. Para evitar dobletes gráficos, creemos que es más conveniente transcribir [ts] siempre como s, sea en posición inicial como interior.

Aunque el fonema / $\theta$ / solo forma parte del sistema fonológico de una minoría de hispanohablantes (Navarro Tomás, 2004 [1918]: 93-95; Lapesa, 2005 [1981]: 487-488, 541-544; Quilis, 2006 [1993]: 283-286; Hualde, 2005: 153-154; RAE, 2010: 123; 2011: 164-168), nosotros lo tendremos presente porque es similar al sonido japonés y porque los seseantes, al leer el japonesismo transcrito en español, siempre podremos pronunciar los alófonos de nuestra s dental. Asimismo, 
como tendremos ocasión de comprobar, de no seguir procedimiento, cargaríamos el grafema s con demasiadas equivalencias fónicas. Según la RAE (2010: 123-125), el fonema / $\theta /$ puede representarse mediante $z$ (ante las vocales/a/,/o/ y /u/) y c (ante/e/, /i/). Existen algunas excepciones para $z$, que a veces se utiliza ante /e/,/i/ para representar "cultismos griegos, arabismos y préstamos de otras lenguas que contienen esta letra en su grafía originaria o en su transcripción al alfabeto latino, así como de algunas voces onomatopéyicas" (RAE, 2010: 124). En nuestra metodología de transcripción, que evita el empleo de dobletes gráficos y pugna por la simplificación, seguiremos el patrón característico del sistema gráfico del español para la representación de los japonesismos que contengan /z/, es decir, transcribiremos z + /a o u/ y c + /e i/32.

En cuanto a /d/, su lugar coronal y su modo de articulación eran indeterminados, por lo que sus realizaciones podían variar: esta unidad rótico-lateral japonesa contaba con un campo de dispersión que incluía realizaciones cercanas a nuestra rótica, alveolar, percusiva [r] y nuestra alveolar, lateral, [I]. Podríamos, pues, transcribirla usando tanto nuestra r como nuestra I, sin embargo, para simplificar, solo emplearemos la grafía $r$ - utilizada en los sistemas de transcripción Hepburn y Kunrei- Tanto en inicial de palabra, como tras un sonido nasal, esta grafía se pronunciará en español [r], esto es, vibrante, alveolar, sonora (como en «requi»). En posición intervocálica, transcrita como r, se realizará como nuestra rótica, alveolar, percusiva, [r] (en japonesismos como: «carate», «nori» o «jaraquiri»).

\subsubsection{Los fonemas dorsales}

El conjunto de consonantes dorso-velares del japonés está constituido por dos fonemas: /k/y/g/, que, tras ser comparadas con las consonantes españolas, presentan las siguientes semejanzas:

\begin{tabular}{|c|c|c|c|c|}
\hline FONEMA & [ \pm SONORO] & [ \pm NASAL] & [ \pm OCLUSIVO] & [ \pm VELAR] \\
\hline /k/ japonés & {$[-]$} & {$[-]$} & {$[+]$} & {$[+]$} \\
\hline /k/ español & {$[-]$} & {$[-]$} & {$[+]$} & {$[+]$} \\
\hline /g/japonés & {$[+]$} & {$[-]$} & {$[+]$} & {$[+]$} \\
\hline /g/ español & {$[+]$} & {$[-]$} & {$[ \pm]^{33}$} & {$[+]$} \\
\hline
\end{tabular}

32 Este patrón actual, según Salvador y Lodares (2008 [1996]: 340-341), es resultado del proceso de simplificación iniciado por la Academia en 1726, de modo que la distribución de las grafías z y c ha continuado esa tendencia hacia la supresión de irregularidades y este rumbo sigue nuestro modelo de transcripción. 
Los fonemas dorsales del japonés se transcribirían de la siguiente manera en español:

\section{TABLA 18}

Adaptación gráfica en español de las unidades dorsales japonesas

\begin{tabular}{c|c}
$\begin{array}{c}\text { FONEMA } \\
\text { JAPONÉS }\end{array}$ & $\begin{array}{c}\text { TRANSCRIPCIÓN GRÁFICA } \\
\text { EN ESPAÑOL }\end{array}$ \\
\hline$/ \mathrm{k} /$ & $\mathrm{C}, \mathrm{c}(+/ \mathrm{a} \circ \mathrm{u} /), \mathrm{Qu}, \mathrm{qu}(+/ \mathrm{e} \mathrm{i} /)$ \\
\hline$/ \mathrm{g} /$ & $\mathrm{G}, \mathrm{g}(+/ \mathrm{a} \mathrm{O} \mathrm{u} /$ ), Gu, gu (+/e i/) \\
\hline
\end{tabular}

En efecto, como aduce la RAE (2010: 116):

La existencia de diversas posibilidades gráficas para representar el fonema/k/ ha dado lugar a numerosos casos de variantes gráficas en la escritura de muchas palabras no pertenecientes al léxico patrimonial. Estas variantes se han generado por la convivencia en el uso de grafías etimológicas con ko q, junto a grafías modificadas para adaptarse al patrón característico del sistema del español, que para representar este fonema emplea la letra c (ante consonante y ante vocales $a, 0, u$ ) o el dígrafo qu (ante las vocales e, $i$ ).

Después ofrece una lista de préstamos con dobletes gráficos y considera preferibles las transcripciones de mayor uso. Así, prefiere caqui a kaki, harakiri a haraquiri, kamikaze a camicace, kimono a quimono, mikado a micado (RAE, 2010: 116-118), a pesar de que más adelante no censure las grafías no recomendadas: "Aunque en muchos casos, especialmente en los préstamos de nueva o más reciente incorporación, el uso mayoritario tiende hoy a conservar los grafemas etimológicos, no pueden considerarse en modo alguno censurables las grafías que se acomoden a las pautas gráficas tradicionales en español” (RAE, 2010: 118).

Obsérvese, asimismo, lo paradójico y aleatorio de aceptar la mayoría de uso en esta ocasión, ya que, por ejemplo, para /ka/ sí es preferible caqui y no kaki, aunque mikado y no micado; para /ki/ prefiere harakiri y no haraquiri, pero sí caqui y no kaki. Con el fin de eliminar esta aleatoriedad e indecisión y enfatizar la sencillez y coherencia con las que pretendemos caracterizar nuestra metodología de transcripción, utilizaremos las pautas gráficas tradicionales en español para adaptar los préstamos procedentes de lengua japonesa a nuestro sistema gráfico. Esta decisión es paralela a la que adoptamos para / $\theta /$. Por tanto, /ka ko ku/ se transcribirán mediante ca, co, cu y /ke ki/ mediante que, qui34.

33 En español puede ser oclusivo o aproximante, dependiendo del contexto.

34 Cierto es que, como sostienen Salvador y Lodares (2008 [1996]: 150-151), la kes una letra de asuntos japoneses o, en otras palabras, una grafía con cualidad de "japonesización”, pero nuestro sistema de transcripción pretende evitar cualquier atributo connotativo para con las grafías empleadas. Por este motivo y por los expuestos en el texto, evitaremos el uso de la letra $k$. 
En cuanto a /g/, según leemos en la RAE (2010: 100-101), "el uso de la letra g o del dígrafo gu para representar el fonema /g/ depende del contexto [...], de cuál sea el fonema siguiente”. Así, los japonesismos se adecuarán a la peculiaridad de nuestro sistema gráfico como si de una palabra patronímica se tratara, esto es, transcribiremos el fonema $/ \mathrm{g} /$ como g ante /a o u/, y como gu ante /e i/, paralelamente a como acabamos de proponer para/k/. De este modo, mientras que los preceptos ortográficos de la Academia prefieren «haikú», «ikebana», «kendo» o «geisha», nuestro método emplea «jaicú», «iquebana», «quendo»o «guesia».

\subsubsection{El fonema laríngeo}

A priori, parece que la unidad laríngea, fricativa, japonesa no se corresponde con ningún fonema similar en español, por lo que únicamente podríamos compararla con un cero fonético. No obstante, la adaptación de dicho sonido a la lengua española se muestra heterogénea, pues la información fónica proporcionada por algunas obras lexicográficas españolas revela que en algunos japonesismos los hablantes han identificado, de un lado, la aspiración laríngea del japonés con nuestra unidad dorsal, fricativa, sorda, /X/, y, de otro, con una aspiración de carácter extranjerizante. Asimismo, encontramos algún ejemplo donde no se produce articulación alguna. De este modo, el grafema $h$-inicial de los siguientes japonesismos ${ }^{35}$ puede representar bien el elemento dorsal /x/: «haicay» (Diccionario del español actual), «haicú» (Diccionario del español actual y Diccionario de uso del español -Moliner, 2008 [1996] —) o «haraquiri» (el Diccionario del español actual y el Gran diccionario de uso del español actual indican que su realización [x] es rara y el Diccionario Clave - Almarza y otros, 2012- señala que es incorrecta); bien la aspiración: «haicay» (Diccionario Clave), «haicú» (Diccionario Clave) o «haraquiri» (Diccionario Clave); o bien la ausencia de articulación: «haraquiri» (Diccionario del español actual, Gran diccionario de uso del español actual y Diccionario de uso del español).

Una vez más, el desinterés al que se han visto sometidos los japonesismos ha provocado que en español sea posible la convivencia de tres soluciones, las cuales revisaremos a continuación para decantarnos por la más adecuada. En primer lugar, desechamos la posibilidad de cero fonético, puesto que en japonés existe una sustancia fónica, que en español es tenida en cuenta la mayoría de las veces, esto es, son más comunes las formas /xaikái/, /xaikú/, / xarakíri/ y sus variantes con elemento aspirado, [h] o [h], que la ausencia de sonido: solo hemos registrado el caso de «haraquiri» (Diccionario del español actual, Gran diccionario de uso del español actual y Diccionario de uso del español), con $h$ muda.

35 Existen otros dos japonesismos, «jentay» y «jiragana», pero de estos no hallamos descriptores fonéticos en las obras lexicográficas españolas. 
Nos restan, pues, dos posibilidades: por un lado, adaptar la aspiración originaria a nuestro / $\mathrm{x}$ / y grafiar el resultado mediante $j$-delante de /a e i o u/ - o g — ante /e/ o /i/_- (RAE, 2010: 101) 0, por otro, utilizar la letra $h$, considerada en nuestro sistema de escritura como un grafema especial, único carácter de nuestro abecedario que no representa ningún fonema en español estándar. Acerca de este elemento gráfico, que "se ha mantenido en nuestro sistema ortográfico por razones etimológicas o de uso tradicional consolidado" (RAE, 2010: 142), aparece en buena parte de préstamos lingüísticos, en los que se usa para representar un sonido aspirado de la lengua de origen (RAE, 2010: 150, 152). En su mayoría, las haches no se pronuncian, pero en los préstamos más recientes se puede conservar esa aspiración (RAE, 2010: 149). Así pues, aunque tal adaptación haya supuesto un procedimiento regular a lo largo del tiempo (Salvador y Lodares, 2008 [1996]: 114), la letra $h$ parece estar adquiriendo en la escritura cierto valor exógeno, al usarse como grafema para marcar la aspiración del préstamo léxico en la lengua originaria. Sin embargo, ya que hemos empleado para nuestro método de transcripción solo grafemas ampliamente asentados en la tradición española, con carga patrimonial, creemos que la elección más lógica para la transcripción del sonido laríngeo del japonés es la letra ${ }^{36}$, con la que grafiamos el sonido velar, fricativo, sordo, [X], además de sus variantes aspiradas meridionales, [h], [h], [h] -entre otras-. Nuestro proceder no resulta tan sorprendente si tenemos en cuenta que algunos extranjerismos y palabras patrimoniales pueden escribirse tanto con $h$ como con $j$ (RAE, 2010: 150, 152). De modo que transcribiremos «jaicay», «jaicú», «jaraquiri», «jentay», «jiragana», etc.

\section{TABLA 19}

Comparación de los rasgos distintivos entre la unidad laríngea japonesa y su española más próxima

\begin{tabular}{c|c|c|c|c} 
FONEMA & {$[ \pm$ SONORO] } & {$[ \pm$ FRICATIVO] } & {$[ \pm$ LARINGEO] } & {$[ \pm$ DORSAL] } \\
\hline /h/japonés & {$[-]$} & {$[+]$} & {$[+]$} & \\
\hline /X/ español & {$[-]$} & {$[+]$} & & {$[+]$} \\
\hline
\end{tabular}

Antes de acabar con este apartado, quisiéramos comentar un aspecto relacionado con el alófono japonés de la combinación /hu/, esto es, [фu]. En español contamos con un fonema labiodental, fricativo, /f/, cuyos alófonos comparten cierta similitud con el sonido japonés, bi-

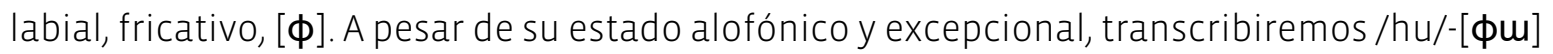
mediante $f u$, ya que refleja, por un lado, una pronunciación similar a la original y, por otro, se encuentra extendido su uso en préstamos y antropónimos: futón, monte Fuji, Fujimura, Fujimoto, Fukuoka, Fukushima, etc.

36 Eliminamos la posibilidad de emplear ge o gi para/xe/y /xi/, respectivamente, con la única finalidad de evitar mayor complejidad a nuestro sistema. 


\section{TABLA 20}

Adaptación gráfica en español de la unidad laríngea japonesa

\begin{tabular}{c|c}
$\begin{array}{c}\text { FONEMA } \\
\text { JAPONÉS }\end{array}$ & $\begin{array}{c}\text { TRANSCRIPCIÓN GRÁFICA } \\
\text { EN ESPAÑOL }\end{array}$ \\
\hline$/ \mathrm{h} /$ & $\mathrm{J}, \mathrm{j}(+/ \mathrm{a}$ e i $/ /), \mathrm{F}, \mathrm{f}(+/ \mathrm{u} /)$ \\
\hline
\end{tabular}

\subsubsection{Unidades consonánticas con palatalización}

Sabemos que el fenómeno de la palatalización afecta al sistema fonológico del japonés, pero no ocurre así en español. Desde un punto de vista articulatorio, la palatalización consiste en la articulación secundaria que tiene lugar cuando la lengua adopta la forma para producir [i], mientras articula un elemento de carácter consonántico. Así, dado que fonológicamente no existen unidades similares en español, trataremos de reproducir gráficamente el material fonético de las unidades palatalizadas mediante la unión de dos grafemas: el primero, que indica el correlato fonológico no palatalizado y, el segundo, la adición de i como elemento de palatalización ${ }^{37}$. Por tanto, la palatalización se reflejaría del siguiente modo:

\section{TABLA 21}

Adaptación gráfica en español de las consonantes palatalizadas japonesas

\begin{tabular}{cc}
$\begin{array}{c}\text { FONEMA } \\
\text { JAPONÉS }\end{array}$ & $\begin{array}{c}\text { TRANSCRIPCIÓN GRÁFICA } \\
\text { EN ESPAÑOL }\end{array}$ \\
\hline$/ \mathrm{p}^{\prime} /$ & $\mathrm{Pl}, \mathrm{pi}$ \\
\hline$/ \mathrm{b}^{\prime} /$ & $\mathrm{BI}, \mathrm{bi}$ \\
\hline$/ \mathrm{m}^{\prime} /$ & $\mathrm{MI}, \mathrm{mi}$ \\
\hline$/ \mathrm{t}^{\prime} /$ & $\mathrm{CH}, \mathrm{ch}$ \\
\hline$/ \mathrm{s}^{\prime} /$ & $\mathrm{SH}, \mathrm{sh} ; \mathrm{Sl}, \mathrm{si}$ \\
\hline$/ \mathrm{z}^{\prime} /$ & $\mathrm{Y}, \mathrm{y}$ \\
\hline$/ \mathrm{d}^{\prime} /$ & $\mathrm{RI}, \mathrm{ri}$ \\
\hline$/ \mathrm{n}^{\prime} /$ & $\tilde{\mathrm{N}}, \tilde{\mathrm{n}}$ \\
\hline$/ \mathrm{k}^{\prime} /$ & $\mathrm{QUI}, \mathrm{qui}$ \\
\hline$/ \mathrm{g}^{\prime} /$ & $\mathrm{GUI}, \mathrm{gui}$ \\
\hline$/ \mathrm{h}^{\prime} /$ & $\mathrm{Jl}, \mathrm{ji}$ \\
\hline
\end{tabular}

37 Esto da lugar en español a que, o bien el elemento palatal $i$ constituye núcleo silábico, o bien se forma un diptongo creciente, donde el primer elemento es una semiconsonante [j-]. Por ejemplo, tanto [p'] como [p'i] se transcriben usando pi, mientras que [p'a] se escribe como pia. 
En primer lugar, se detectan solamente cuatro casos excepcionales: las unidades coronales palatalizadas /t'/, /s'/, /z'/ y /n'/, es decir, para su representación gráfica, no utilizamos un grafema consonántico español seguido de $i$, sino que se emplean letras mediante las que reflejamos elementos palatales en nuestra lengua que comparten una gran similitud fonética con las realizaciones japonesas.

De este modo, para el caso de /t'/ utilizamos el dígrafo ch, por compartir ambos elementos una gran cantidad de rasgos fonéticos:

\section{TABLA 22}

Comparación de los rasgos distintivos entre la coronal japonesa /t’’y y su española más próxima

\begin{tabular}{c|c|c|c|c} 
FONEMA & {$[ \pm$ SONORO $]$} & {$[ \pm$ NASAL $]$} & {$[ \pm \text { OCLUSIVO }]^{38}$} & {$[ \pm$ CORONAL $]$} \\
\hline$/ t^{\prime} /$ japonés & {$[-]$} & {$[-]$} & {$[+]$} & {$[+]$} \\
\hline /ty/español & {$[-]$} & {$[-]$} & {$[+]$} & {$[+]$} \\
\hline
\end{tabular}

En cuanto a / $z$ '/, emplearemos el grafema $y$, representante de un sonido palatal, fricativo y sonoro, muy próximo a las realizaciones japonesas de /z'/:

\section{TABLA 23}

Comparación de los rasgos distintivos entre la coronal japonesa /z’/ y su española más próxima

\begin{tabular}{c|c|c|c|c} 
FONEMA & {$[ \pm$ SONORO $]$} & {$[ \pm$ NASAL $]$} & {$[ \pm$ FRICATIVO] } & {$[ \pm$ CORONAL] } \\
\hline$/ z^{\prime} /$ japonés & {$[+]$} & {$[-]$} & {$[+]$} & {$[+]$} \\
\hline /d/ español & {$[+]$} & {$[-]$} & {$[+]^{39}$} & {$[+]$} \\
\hline
\end{tabular}

En la actualidad, "la gran mayoría de los hispanohablantes no distinguen ya en la pronunciación de los fonemas /y/ y /II/, que se han reducido a uno solo, el fonema /y/, dando lugar al fenómeno conocido como yeísmo" (RAE, 2010: 120). Por este motivo, resulta imposible "determinar gracias a la pronunciación si una palabra ha de escribirse con y o con II, por lo que únicamente la consulta al diccionario permite resolver, en última instancia, las dudas que a este respecto puedan planteársele a quien escribe" (RAE, 2010: 120). Para evitar dobletes gráficos y por coherencia con el yeísmo, fenómeno prácticamente mayoritario, usaremos siempre

38 El rasgo distintivo de estas unidades era la oclusión, aunque desde un punto de vista fonético sean realizaciones africadas.

39 Este fonema presenta realizaciones africadas, lo que significa que siempre tiene un momento fricativo. 
la letra $y$. Ante voces transcritas como ninja, dojo, judo, jiu-jitsu40 o ginkgo, nuestro método propone «ninya», «doyo», «yudo», «yuyisu»o «yinco».

Por otro lado, quisiéramos describir la situación de /s'. Hemos empleado para su transcripción dos tipos de grafemas: sh y si. Ya mencionamos que este sonido no existe en español actual y que puede ser representado mediante sh (transcribiendo el préstamo en cursiva) o con los valores fónicos de s o ch (RAE, 2010: 127). Para no confundirlo con la representación gráfica del fonema japonés /t'/, que transcribimos mediante ch, y para no sobrecargar el grafema s con demasiados valores fónicos, deberíamos emplear sh y transcribir el vocablo en cursiva, o emplear la grafía si. Dado que tratamos de eliminar cualquier atisbo de doblete gráfico, creemos mejor opción transcribir siempre el fonema japonés/s'/ mediante el dígrafo si. De esta forma, evitamos un elemento extraño y perturbador de nuestro sistema de transcripción. Preferimos «busido» a «bushido», «guesia»a «geisha», «sasimi» a «sashimi» 0 «siamisén» a «shamisén».

En último lugar, utilizaremos dos grafemas, ñ y n, para la representación del fonema /n’\% Sabemos que esta unidad puede aparecer tanto en posición explosiva como implosiva. Para el primer caso de distribución utilizaremos la letra ñ, mientras que para el segundo, con un amplio abanico de realizaciones, emplearemos no m - según el contexto-, pues el fonema representado gráficamente por ñ jamás aparece en posición implosiva en español (Alarcos, 1986 [1965]: 182; Martínez Celdrán, 1989: 50-51; Navarro Tomás, 2004 [1918]: 133; Quilis, 2006 [1993]: 226; RAE, 2010: 239). Así, pues, usaremos «tanca», «ninya», «cen» 0 «ien», entre otras. Véase la tabla comparativa de tales elementos:

\section{TABLA 24}

Comparación de los rasgos distintivos entre la coronal japonesa /n’/ y su española más próxima

\begin{tabular}{ccccc} 
FONEMA & {$[ \pm$ SONORO $]$} & {$[ \pm$ NASAL $]$} & {$[ \pm$ CORONAL] } \\
$/ \mathrm{n}^{\prime} /$ japonés & {$[+]$} & {$[+]$} & {$[+]$} \\
\hline$/ \mathrm{n} /$ español & {$[+]$} & {$[+]$} & {$[+]$} \\
\hline
\end{tabular}

En conclusión, casi todas las unidades consonánticas palatalizadas quedarán transcritas mediante la combinación gráfica de consonante $+i^{41}$. Dentro de este grupo, únicamente ha-

40 Recuérdese que j se usa para transcribir la aspirada laríngea japonesa, /h/.

41 Hemos de advertir que las unidades palatalizadas seguidas del sonido vocálico [i] ofrecen una doble posibilidad de transcripción: por ejemplo, tanto /p'/ como /p'i/ se transcriben mediante pi. Sin embargo, jamás aparecen sin acompañamiento vocálico, por lo que esta característica no nos ha de preocupar para nuestro método de transcripción. 
Ilamos cierta anomalía en qui, y gui, pues la tradición gráfica española pide que las combinaciones /ki/ y /gi/ requieran un dígrafo.

\section{TABLA 25}

Adaptación gráfica — casi regular - en español de las consonantes palatalizadas japonesas

\begin{tabular}{c|c}
$\begin{array}{c}\text { FONEMA } \\
\text { JAPONÉS }\end{array}$ & $\begin{array}{c}\text { TRANSCRIPCIÓN } \\
\text { GRÁFICA EN ESPAÑOL }\end{array}$ \\
\hline$/ \mathrm{p}^{\prime} /$ & $\mathrm{Pl}, \mathrm{pi}$ \\
\hline$/ \mathrm{b}^{\prime} /$ & $\mathrm{Bl}, \mathrm{bi}$ \\
\hline$/ \mathrm{m}^{\prime} /$ & $\mathrm{Ml}, \mathrm{mi}$ \\
\hline$/ \mathrm{s}^{\prime} /$ & $\mathrm{SI}, \mathrm{si}$ \\
\hline$/ \mathrm{d}^{\prime} /$ & $\mathrm{RI}, \mathrm{ri}$ \\
\hline$/ \mathrm{k}^{\prime} /$ & $\mathrm{QUI}, \mathrm{qui}$ \\
\hline$/ \mathrm{g}^{\prime} /$ & $\mathrm{GUI}, \mathrm{gui}$ \\
\hline$/ \mathrm{h}^{\prime} /$ & $\mathrm{Jl}, \mathrm{ji}$ \\
\hline
\end{tabular}

Por otro lado, existen tres unidades consonánticas palatalizadas que utilizan otras grafías sin i, puesto que marcan mejor su naturaleza palatalizada: «ninya» o «nunchaco».

\section{TABLA 26}

Adaptación gráfica -irregular - en español de las consonantes palatalizadas japonesas

\begin{tabular}{c|c}
$\begin{array}{c}\text { FONEMA } \\
\text { JAPONÉS }\end{array}$ & $\begin{array}{c}\text { TRANSCRIPCIÓN GRÁFICA } \\
\text { EN ESPAÑOL }\end{array}$ \\
\hline$/ \mathrm{t}^{\prime} /$ & $\mathrm{CH}, \mathrm{ch}$ \\
\hline$/ \mathrm{Z}^{\prime} /$ & $\mathrm{Y}, \mathrm{y}$ \\
\hline$/ \mathrm{n}^{\prime} /$ & $\tilde{\mathrm{N}}, \tilde{\mathrm{n}}(\mathrm{N} \circ \mathrm{M}, \mathrm{n} \circ \mathrm{m}$-implosiva-) \\
\hline
\end{tabular}

\subsection{La geminación de las unidades consonánticas}

Al igual que el sistema vocálico, el sistema consonántico del japonés cuenta con la oposición entre unidades largas o geminadas, que se oponen a sus respectivos correlatos simples (Smith, 1980; Vance, 1987 y 2008; Rodríguez-Izquierdo, 1991; Akamatsu, 1997 y 2000; Grenon, 2005; Ito, Kang y Kenstowicz, 2006; Tsujimura, 2007 [1996]; Kubozono, Itô y Mester, 2009; FreIlesvig, 2010; Labrune, 2012). Akamatsu (1997: 154; 2000: 195-196), Kubozono, Itô y Mester (2009: 2-4) y Labrune (2012: 104, 136) convienen en destacar varias características para las unidades consonánticas geminadas. En primer lugar, solo aparecen en posición intervocálica. Por otro lado, los estudios fonéticos revelan que la longitud consonántica está señalada principal- 
mente por la duración consonántica, pero no por cambios cualitativos. Asimismo, no todas las unidades no vocálicas pueden ser geminadas y, de tener lugar, no significa que se opongan a un correlato simple. Por tanto, en conexión con el punto anterior, el japonés, como otras muchas lenguas (Ladefoged y Maddieson, 1996: 92), presenta una restricción en cuanto a las unidades que pueden ser geminadas. En último lugar, los elementos geminados no pueden aparecer después de una vocal larga o combinación vocálica. Así, por ejemplo, 時 [tok’i] 'tiempo', 突起 [tokk'i] ‘saliente, protuberancia’ y 陶器 [to:k'i] 'cerámica' son formas válidas, pero no *[to:kk'i].

Según Labrune (2012: 136), solo algunas unidades consonánticas sordas /p/, /p’/, /t/, /t’/, $/ \mathrm{k} /, / k^{\prime} /, / \mathrm{s} / \mathrm{y} / \mathrm{s}^{\prime} /$ tienden a la geminación en el léxico patrimonial del japonés, lo cual es prácticamente imposible para las sonoras, que no geminan en el estrato fonológico perteneciente al léxico patrimonial (Labrune, 2012: 104). Sin embargo, como sostiene Labrune (2012: 104), sí se pueden hallar algunos pocos ejemplos de consonantes sonoras en el léxico relacionado con la mimética u onomatopeya (ざっぶんと [zabbunto] 'con un gran splash') y los préstamos occidentales (バッグく bag [baggm] 'bolso', ベッドく bed [beddo] 'cama'), muchos de los cuales conviven con las variantes sordas (バッグ < bag [bakkm] ‘bolso', ベット < bed [betto] 'cama’). En cuanto a las versiones geminadas de / ////l'/,/j/ y / w/ y /h/-/h'/, son casi inexistentes, exceptuando algunos ejemplos marginales, tales como préstamos recientes o palabras onomatopéyicas: バッハ [bahha] 'Bach', 十針 [z'whhal'i] 'diez puntos', ウッフウッフ/uhhuhhu/ expresar risa ‘ja, ja, ja' (Labrune, 2012: 136). La marginalidad con respecto al resto de elementos operativos dentro del sistema lingüísticos japonés demuestra que el conjunto de aproximantes centrales (/ ل l' j u/) y laríngeos (/h h'/) no posee esta cualidad distributiva entre sus componentes².

Para la geminación, baste decir que en español, salvo en pocos casos, no admite la geminación de los fonemas consonánticos (Alarcos, 1986 [1965]: 186-195; Hidalgo y Quilis, 2004: 170-171; Quilis, 2006 [1993]: 381-383; RAE, 2010: 174)43. De modo que la duplicación de los fonemas consonánticos del japonés no tendrá reflejo en la grafía, esto es, no duplicaremos los grafemas consonánticos para representar el fenómeno de la geminación ${ }^{44}$. En realidad, en el

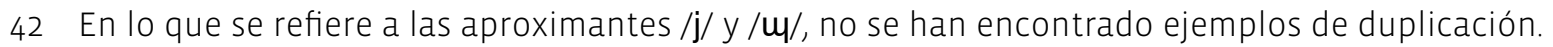
En cuanto a /d/, solo descubrimos un único ejemplo con función expresiva en Labrune (2012: 136): [ballabala] 'en pedazos, en trozos'. Al igual que en el ejemplo con /h/ que recogimos de Labrune, Akamatsu (1997: 154) aporta dos únicos ejemplos, otros extranjerismos: マッハ [mahha]< Mach, 'la velocidad de una aeronave' y スタッブ く staff [sutahhu] 'personal'.

43 RAE (2010: 177): «Las dos únicas letras que pueden aparecer duplicadas en palabras españolas representando la pronunciación doble o geminada de un mismo fonema consonántico son la $n$ $(-n n-)$ y la $b(-b b-))$.

44 Como hemos podido comprobar en los ejemplos proporcionados por Labrune (2012: 104), en lengua japonesa, la duplicación consonántica se refleja mediante los cana つ —en jiragana—y ツ - catacana- reducidos. 
proceso de asimilación gráfica de los extranjerismos, el español peninsular tiende a simplificar las consonantes dobles, por lo que nuestra resolución atiende, además, a dicha tendencia: spaghetti > espagueti, buffet > bufé / bufet, scanner > escáner (Gómez Capuz, 2005: 19).

\section{Conclusiones}

Habiendo examinado a fondo los sistemas gráfico-fonológicos de ambos sistemas lingüísticos, podemos resumir en la siguiente tabla nuestro método de transcripción de las unidades vocálicas, semivocálicas y consonánticas.

Ensombrecimos los casos de: 1) transcripción de grafías obsoletas o con lectura irregular en la propia lengua japonesa (ui de/ui/ y ue de /ue/ y o de un pretérito /uo/, cuya pronunciación actual es /o//); 2) transcripciones que requieren una letra diferente al resto del paradigma (su para /tu/ o fu para /hu/); 3) transcripciones que usan el mismo grafema para diferentes paradigmas (su de /tu/ y su de/su/).

\section{TABLA 27}

Nuestro sistema de transcripción de las unidades vocálicas japonesas

\begin{tabular}{|c|c|c|c|}
\hline & $\begin{array}{l}\text { SILABARIO } \\
\text { JIRAGANA }\end{array}$ & $\begin{array}{l}\text { SILABARIO } \\
\text { CATACANA }\end{array}$ & $\begin{array}{l}\text { TRANSCRIPCIÓN } \\
\text { EN ESPAÑOL }\end{array}$ \\
\hline$/ \mathrm{a} /$ & あ & ア & $\mathrm{A}, \mathrm{a}$ \\
\hline /i/ & い & 1 & $\mathrm{I}, \mathrm{i}$ \\
\hline /u/ & う & ウ & $U, u$ \\
\hline /e/ & え & 工 & $E, e$ \\
\hline $10 /$ & お & オ & 0,0 \\
\hline
\end{tabular}

\section{TABLA 28}

Nuestro sistema de transcripción de las unidades semivocálicas japonesas

\begin{tabular}{|c|c|c|c|c|c|c|c|c|c|c|c|}
\hline \multirow[b]{2}{*}{ /j/ } & \multicolumn{5}{|c|}{ SILABARIO JIRAGANA } & \multicolumn{5}{|c|}{ SILABARIO CATACANA } & \multirow{2}{*}{$\begin{array}{l}\text { TRANSCRIPCIÓN } \\
\text { EN ESPAÑOL } \\
\text { IA, ia, IU, } \\
i u, I 0, i o .\end{array}$} \\
\hline & $\begin{array}{c}や \\
{[j a]}\end{array}$ & -- & $\begin{array}{c}\text { ゆ } \\
{[j m]}\end{array}$ & -- & $\begin{array}{c}\text { よ } \\
\text { [jo] }\end{array}$ & $\begin{array}{c}\text { P } \\
{[\mathrm{ja}]}\end{array}$ & -- & $\begin{array}{c}\text { 그 } \\
\text { [jw] }\end{array}$ & -- & $\begin{array}{c}\exists \\
{[j o]}\end{array}$ & \\
\hline$/ щ /$ & $\begin{array}{c}\text { わ } \\
\text { [щa] }\end{array}$ & $\begin{array}{l}\text { み } \\
\text { [ui] }\end{array}$ & -- & $\begin{array}{c}\text { 点 } \\
\text { [ue] }\end{array}$ & $\begin{array}{c}\text { を } \\
{[0]}\end{array}$ & $\begin{array}{c}\text { ワ } \\
\text { [ua] }\end{array}$ & $\begin{array}{c}\text { मे } \\
\text { [uil] }\end{array}$ & -- & $\begin{array}{c}\text { ㄱ } \\
\text { [üe] }\end{array}$ & $\begin{array}{c}7 \\
{[0]}\end{array}$ & $\begin{array}{l}\text { UA, ua, UI, ui, } \\
\text { UE, ue, O, o. }\end{array}$ \\
\hline
\end{tabular}


TABLA 29

Nuestro sistema de transcripción de las unidades consonánticas japonesas

\begin{tabular}{|c|c|c|c|c|c|c|c|c|}
\hline & \multicolumn{4}{|c|}{ SILABARIO JIRAGANA } & \multicolumn{3}{|c|}{ SILABARIO CATACANA } & \multirow{2}{*}{$\begin{array}{l}\text { TRANSCRIPCIÓN } \\
\text { EN ESPAÑOL } \\
\text { PA, pa, PU, pu, } \\
\text { PE, pe, PO, po }\end{array}$} \\
\hline & $\begin{array}{c}\text { ぱ } \\
{[\mathrm{pa}]}\end{array}$ & $\begin{array}{c}30 \\
{[p m]}\end{array}$ & ペ & $\begin{array}{c}\text { ぽ } \\
{[\text { po] }}\end{array}$ & $\begin{array}{c}\text { パ } \\
{[\mathrm{pa}]}\end{array}$ & $-\quad \begin{array}{c}\text { プ } \\
--\quad[p w]\end{array}$ & \begin{tabular}{cc}
$\hat{\mathrm{pe}}^{0}$ & \multirow{2}{*}{} \\
{$[\mathrm{po}]$}
\end{tabular} & \\
\hline$/ p^{\prime} /$ & 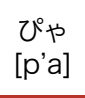 & $\begin{array}{cc}\text { ぴ } & \text { びゅ } \\
\text { [p'i] } & {\left[p^{\prime} m\right]}\end{array}$ & -- & $\begin{array}{l}\text { ぴょ } \\
{\left[p^{\prime} \circ\right]}\end{array}$ & $\begin{array}{l}\text { ピャ } \\
{\left[p^{\prime} a\right]}\end{array}$ & $\begin{array}{cc}ヒ^{\circ} & ヒ^{\circ} \beth \\
{\left[p^{\prime} i\right]} & {\left[p^{\prime} m\right]}\end{array}$ & $\begin{array}{l}\text { ピョ } \\
{\left[p^{\prime} \circ\right]}\end{array}$ & $\begin{array}{l}\text { PIA, pia, PI, pi, } \\
\text { PIU, piu, PIO, pio }\end{array}$ \\
\hline & $\begin{array}{c}\text { ば } \\
{[\mathrm{ba}]}\end{array}$ & $\begin{array}{c}\text { ぶ } \\
{[\mathrm{bum}]}\end{array}$ & べ & $\begin{array}{c}\text { ぼ } \\
{[\text { [bo] }}\end{array}$ & $\begin{array}{c}\text { バ } \\
{[\mathrm{ba}]}\end{array}$ & 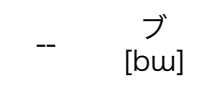 & $\begin{array}{cc}\text { ベ } & \text { ボ } \\
{[\text { be] }} & {[\mathrm{bo}]}\end{array}$ & $\begin{array}{l}\text { BA, ba, BU, bu, } \\
\text { BE, be, BO, bo }\end{array}$ \\
\hline /b'/ & $\begin{array}{l}\text { びゃ } \\
{\left[b^{\prime} a\right]}\end{array}$ & $\begin{array}{cc}\text { び } & \text { びゅ } \\
{\left[b^{\prime} i\right]} & {\left[b^{\prime} m\right]}\end{array}$ & -- & $\begin{array}{l}\text { びょ } \\
{\left[b^{\prime} \circ\right]}\end{array}$ & $\begin{array}{l}\text { ビャ } \\
{\left[b^{\prime} a\right]}\end{array}$ & $\begin{array}{cc}\text { ビ } & \text { ビュ } \\
{\left[b^{\prime} i\right]} & {\left[b^{\prime} w\right]}\end{array}$ & $\begin{array}{l}\text { ビョ} \\
{\left[b^{\prime} \circ\right]}\end{array}$ & $\begin{array}{l}\text { BIA, bia, BI, bi, } \\
\text { BIU, biu, BIO, bio }\end{array}$ \\
\hline /m/ & $\begin{array}{c}\text { ま } \\
\text { [ma] }\end{array}$ & $\begin{array}{c}\text { む } \\
{[\mathrm{mul}]}\end{array}$ & $\begin{array}{c}\text { め } \\
{[\mathrm{me}]}\end{array}$ & $\begin{array}{c}も \\
{[\mathrm{mo}]}\end{array}$ & $\begin{array}{c}\nabla \\
{[\mathrm{ma}]}\end{array}$ & $\begin{array}{c}\Delta \\
{[\mathrm{mm}]}\end{array}$ & $\begin{array}{cc}x & € \\
{[\mathrm{me}]} & {[\mathrm{mo}]}\end{array}$ & $\begin{array}{l}\mathrm{MA}, \mathrm{ma}, \mathrm{MU}, \mathrm{mu} \text {, } \\
\mathrm{ME}, \mathrm{me}, \mathrm{MO}, \mathrm{mo}\end{array}$ \\
\hline$/ \mathrm{m}^{\prime} /$ & $\begin{array}{l}\text { みや } \\
{\left[\mathrm{m}^{\prime} \mathrm{a}\right]}\end{array}$ & $\begin{array}{cc}\text { み } & \text { みゆ } \\
\text { [m'i] } & {\left[m^{\prime} m\right]}\end{array}$ & -- & $\begin{array}{l}\text { みょ } \\
\text { [m'o] }\end{array}$ & $\begin{array}{l}\text { ミャ } \\
{\left[\mathrm{m}^{\prime} \mathrm{a}\right]}\end{array}$ & $\underset{\left[m^{\prime} i\right]}{\equiv} \quad \underset{\left[m^{\prime} u\right]}{\equiv}$ & $\begin{array}{l}\text { ミョ} \\
{\left[\mathrm{m}^{\prime} \mathrm{o}\right]}\end{array}$ & $\begin{array}{l}\text { MIA, mia, MI, } \\
\text { mi, MIU, miu, } \\
\text { MIO, mio }\end{array}$ \\
\hline /t/ & $\begin{array}{c}た \\
{[\mathrm{ta}]}\end{array}$ & $\stackrel{?}{\text { [tsul] }}$ & $\begin{array}{c}\tau \\
{[\mathrm{te}]}\end{array}$ & $\begin{array}{c}\varepsilon \\
\text { [to] }\end{array}$ & $\begin{array}{c}\text { 夕 } \\
{[\mathrm{ta}]}\end{array}$ & $\begin{array}{l}\text { ツ } \\
\text { [tsw] }\end{array}$ & $\begin{array}{c}r \\
\text { [to] }\end{array}$ & $\begin{array}{l}\text { TA, ta, SU, su, } \\
\text { TE, te, TO, to }\end{array}$ \\
\hline /t'/ & $\begin{array}{l}5 ゃ \\
\text { [t'a] }\end{array}$ & $\begin{array}{c}5 \phi \\
{\left[t^{\prime} w\right]}\end{array}$ & -- & $\begin{array}{c}5 ょ \\
\text { [t'o] }\end{array}$ & $\begin{array}{l}\text { チャ } \\
\text { [t'a] }\end{array}$ & $\begin{array}{l}\text { チュ } \\
\text { [t'm] }\end{array}$ & $\begin{array}{l}\text { チョ} \\
\text { [t’o] }\end{array}$ & $\begin{array}{l}\text { CHA, cha, CHI, } \\
\text { chi, CHU, chu, } \\
\text { CHO, cho }\end{array}$ \\
\hline /d/ & $\begin{array}{c}\text { だ } \\
{[\mathrm{da}]}\end{array}$ & - & $\begin{array}{c}\text { で } \\
{[\mathrm{de}]}\end{array}$ & $\begin{array}{c}\text { ぞ } \\
\text { [do] }\end{array}$ & $\begin{array}{c}\text { ダ } \\
\text { [da] }\end{array}$ & -- & $\begin{array}{c}\text { デ } \\
{[\mathrm{de}]}\end{array}$ & $\begin{array}{l}\text { DA, da, DE, de, } \\
\text { DO, do }\end{array}$ \\
\hline$/ s /$ & $\begin{array}{c}\text { さ } \\
{[\mathrm{sa}]}\end{array}$ & $\begin{array}{c}\text { す } \\
{[\mathrm{suw}]}\end{array}$ & $\begin{array}{c}\text { せ } \\
{\left[\begin{array}{c}\mathrm{se} \\
---\end{array}\right.}\end{array}$ & $\begin{array}{c}z \\
z \\
{[\mathrm{so}]}\end{array}$ & $\begin{array}{c}\text { サ } \\
\text { [sa] }\end{array}$ & $\left.\begin{array}{c}x \\
{[\mathrm{sum}}\end{array}\right]$ & $\begin{array}{c}\text { ソ[ } \\
\text { so] } \\
----\end{array}$ & $\begin{array}{l}\text { SA, sa, SU, Su, } \\
\text { SE, se, SO, SO }\end{array}$ \\
\hline$\left|s^{\prime}\right|$ & $\begin{array}{l}\text { しや } \\
\text { [s'a] }\end{array}$ & $\begin{array}{cc}\text { し } & \text { しゅ } \\
\text { [s'i] } & {\left[s^{\prime} w\right]}\end{array}$ & -- & $\begin{array}{l}\text { しょ } \\
{\left[\mathrm{s}^{\prime} \mathrm{o}\right]}\end{array}$ & $\begin{array}{l}\text { シャ } \\
\text { [s'a] }\end{array}$ & $\begin{array}{cc}\text { シ } & \text { シュ } \\
\text { [s'i] } & \text { [s'u] }\end{array}$ & $\begin{array}{l}\text { ショ } \\
{\left[\mathrm{s}^{\prime} \mathrm{O}\right]}\end{array}$ & $\begin{array}{l}\text { SIA, sia, SI, si, } \\
\text { SIU, siu, SIO, sio }\end{array}$ \\
\hline$|z|$ & $\begin{array}{c}\text { ざ } \\
\text { [za] }\end{array}$ & $\begin{array}{l}\text { ず,づ } \\
\text { [zu] }\end{array}$ & $\begin{array}{c}\text { ぜ } \\
{[\mathrm{ze}]}\end{array}$ & $\begin{array}{c}\text { ぞ } \\
\text { [zo] }\end{array}$ & $\begin{array}{c}\text { ザ } \\
\text { [za] }\end{array}$ & $\begin{array}{l}\text { ズ,ヅ } \\
\text { [zw] }\end{array}$ & $\begin{array}{l}\text { ゾ } \\
\text { [zo] }\end{array}$ & $\begin{array}{l}\text { ZA, za, ZU, zu, } \\
C E, c e, Z O, z o\end{array}$ \\
\hline /Z'/ & $\begin{array}{l}\text { じゃ, } \\
\text { ぢゃ } \\
\text { [z'a] }\end{array}$ & $\begin{array}{cc}\text { じ,ぢ } & \text { ப゙ゅ, } \\
\text { [z'i] } & \text { よ゙ゅ } \\
& {\left[z^{\prime} m\right]}\end{array}$ & -- & $\begin{array}{l}\text { じょ, } \\
\text { ぢょ } \\
{\left[z^{\prime} \circ\right]}\end{array}$ & $\begin{array}{l}\text { ジャ, } \\
\text { ヂャ } \\
\text { [z'a] }\end{array}$ & $\begin{array}{cc}\text { ジ, } & \text { ジュ, } \\
\text { ヂ } & \text { ヂュ } \\
\text { [z'i] } & {\left[z^{\prime} m\right]}\end{array}$ & $\begin{array}{l}\text { ジョ, } \\
\text { ヂョ } \\
{\left[\mathrm{z}^{\prime} \mathrm{O}\right]}\end{array}$ & $\begin{array}{l}\text { YA, ya, YI, yi, } \\
\text { YU, yu, YO, yo }\end{array}$ \\
\hline$/ \mathrm{d} /$ & $\begin{array}{c}5 \\
{[1 a]}\end{array}$ & $\begin{array}{c}\text { る } \\
{[\mathrm{lu}]}\end{array}$ & $\begin{array}{c}れ \\
{[l e]}\end{array}$ & $\begin{array}{c}3 \\
{[10]}\end{array}$ & $\begin{array}{l}\text { ラ } \\
{[\mathrm{da}]}\end{array}$ & $\begin{array}{l}\text { ル } \\
{[\lambda w]}\end{array}$ & $\begin{array}{c}\text { ㅁ } \\
{[10]}\end{array}$ & $\begin{array}{l}\text { RA, ra, RU, ru, } \\
\text { RE, re, RO, ro }\end{array}$ \\
\hline$/ d^{\prime} /$ & $\begin{array}{l}\eta_{\Perp} \\
{\left[l^{\prime} a\right]}\end{array}$ & $\begin{array}{cc}\eta & \eta \Phi \\
{\left[l^{\prime} i\right]} & {\left[l^{\prime} m\right]}\end{array}$ & -- & $\begin{array}{l}\text { りょ } \\
\text { [l'o] }\end{array}$ & $\begin{array}{l}\text { リャ } \\
\text { [l'a] }\end{array}$ & $\begin{array}{cc}\text { リ } & \text { リュ } \\
\text { ['li] } & \text { [l'm] }\end{array}$ & $\begin{array}{l}\text { リョ } \\
{[\text { [’’o] }}\end{array}$ & $\begin{array}{l}\text { RIA, ria, RI, ri, } \\
\text { RIU, riu, RIO, rio }\end{array}$ \\
\hline /n/ & $\begin{array}{c}\text { な } \\
{[\mathrm{na}]}\end{array}$ & $\begin{array}{c}ぬ \\
{[n w]}\end{array}$ & $\begin{array}{c}\downarrow \\
{[n e]}\end{array}$ & $\begin{array}{l}\text { の } \\
{[\mathrm{no}]}\end{array}$ & $\underset{[\mathrm{na}}{+}$ & $\begin{array}{c}\text { ヌ } \\
{[n w]}\end{array}$ & [no] & $\begin{array}{l}\text { NA, na, NU, nu, } \\
N E \text {, ne, No, no }\end{array}$ \\
\hline$/ n^{\prime}-1$ & $\begin{array}{l}\text { にゃ } \\
\text { [n'a] }\end{array}$ & $\begin{array}{cc}k & \text { に中 } \\
{\left[n^{\prime} i\right]} & {\left[n^{\prime} w\right]}\end{array}$ & - & $\begin{array}{l}\text { にょ } \\
{\left[n^{\prime} \circ\right]}\end{array}$ & $\begin{array}{l}\text { ニャ } \\
\text { [n'a] }\end{array}$ & $\underset{\left[n^{\prime} i\right]}{\overline{\text { nㅡㄹ }}}$ & $\begin{array}{l}=\Xi \\
{\left[n^{\prime} \circ\right]}\end{array}$ & $\begin{array}{l}\tilde{N} A, \tilde{n} a, \tilde{N} I, \text { ñi, } \\
\tilde{N} U, \tilde{n} u, \tilde{N} O, \tilde{n} O\end{array}$ \\
\hline
\end{tabular}




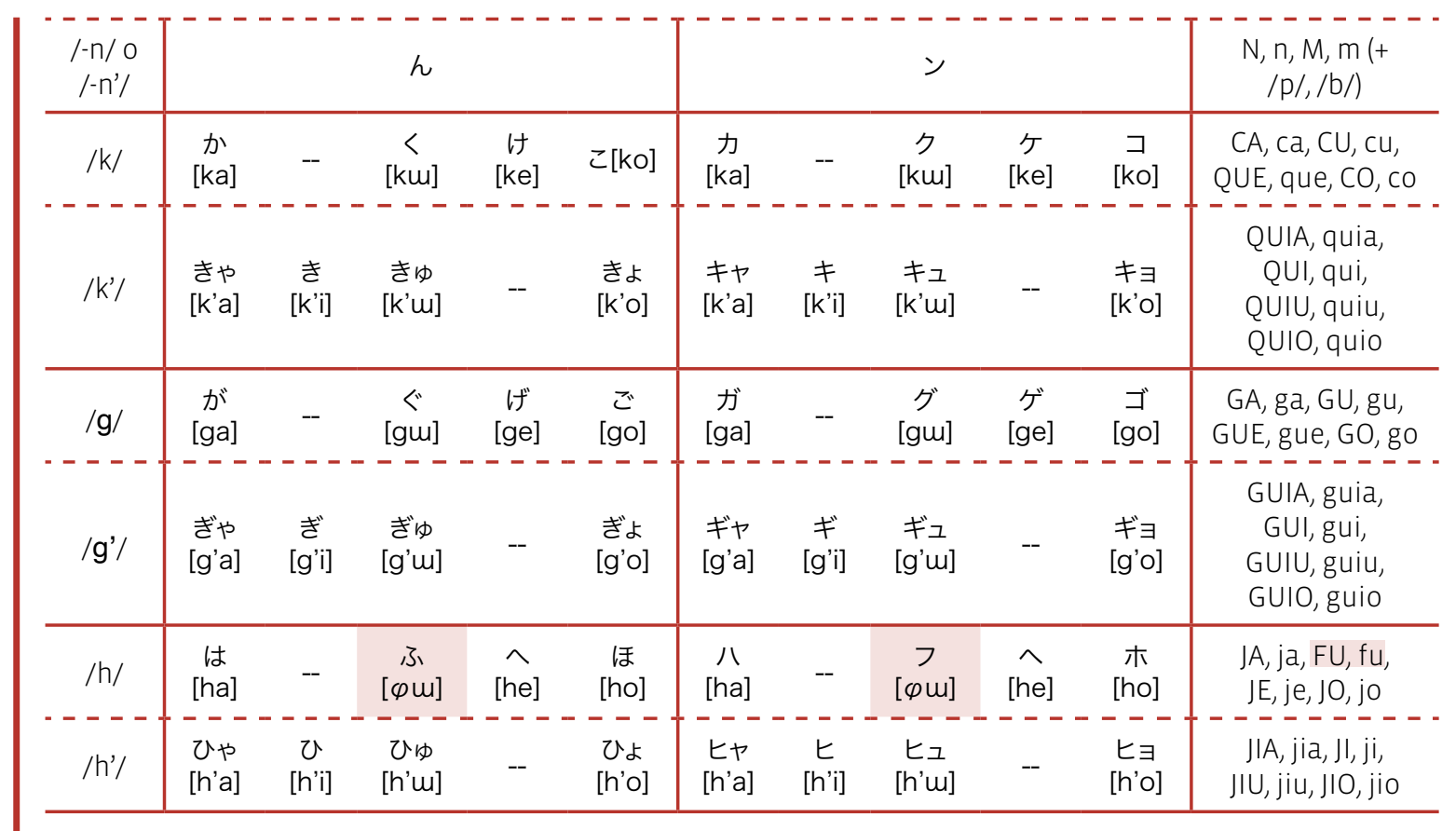

\section{Referencias bibliográficas}

Abercrombie, Daniel Jones, 1967: Elements of General Phonetics, Chicago: Aldine Atherton.

Akamatsu, Tsutomu, 1997: Japanese Phonetics: Theory and Practice, Múnich: LINCOM Europa.

Akamatsu, Tsutomu, 2000: Japanese Phonology: A Functional Approach, Múnich: LINCOM Europa.

Alarcos Llorach, Emilio, 1986 [1965]: Fonología española, Madrid: Gredos.

Almarza, Nieves, y otros, 2012: Diccionario Clave: diccionario de uso del español actual, Madrid: S.M. [http://clave.smdiccionarios.com/app.php, fecha de consulta: 27 de mayo de 2016].

Canellada, María Josefa, y John Kuhlmann Madsen, 1987: Pronunciación del español: lengua hablada y literaria, Madrid: Castalia.

Crawford, Clifford James, 2009: "Adaptation and Transmission in Japanese Loanword Phonology". Tesis de doctorado, University of Cornell: EEUU [http://ecommons.library.cornell.edu/ bitstream/1813/13947/1/Crawford,\%20Clifford.pdf, fecha de consulta: 27 de mayo de 2016].

Del Moral, Rafael, 2002: Diccionario Espasa: Lenguas del mundo, Madrid: Espasa.

D'introno, Francesco, Enrique del Teso y Rosemary Weston, 1995: Fonética y Fonología actual del español, Madrid: Cátedra. 
Fernández Mata, Rafael, 2015: "Estudio histórico del doblete catán-catana en lengua española”, Revista de Lexicografía XXI, 17-30.

Fernandez Mata, Rafael, 2016: Los japonesismos de la lengua española: Historia y transcripción. Tesis inédita, Universidad Pablo de Olavide, Sevilla.

Fernández Mata, Rafael, 2017: “Los japonesismos del español actual”, Revista de Filología de la Universidad de La Laguna 35, 149-168.

Ferres SerRano, Juan José, 2001: Gunkán, Diccionario de Kanjis Japoneses, Madrid: Hiperión.

Frellesvig, Bjarke, 2010: A History of the Japanese Language, Cambridge: Cambridge University Press.

Gil Fernández, Juana, 2007: Fonética para profesores de español: de la teoría a la práctica, Madrid: Arco, D. L.

Gómez Capuz, Juan, 2005: La inmigración léxica, Madrid: Arco Libros.

Gottlıeb, Nanette, 2010: "The Rōmaji movement in Japan”, Journal of the Royal Asiatic Society (Third Series) 20 (1), 75-88.

Grenon, Izabelle, 2005: "The status of the sound [z] in Japanese", Langues et Linguistique 31, 63-90.

Hara, Makoto, 1994: "Método de enseñanza de la pronunciación española a los Alumnos japoneses" en Salvador Montesa Peydró y Antonio Garrido Moraga (eds.): Actas del Segundo Congreso Nacional de ASELE. Español para extranjeros: Didáctica e Investigación, Málaga: ASELE, 371-380.

Hasegawa, Yoko, 1999: "Pitch accent and vowel devoicing in Japanese" en J. Ohala, M. Ohala, D. Granville y A. Bailey (eds.): Proceedings of the 14th International Congress of Phonetic Sciences, Berkeley: Universidad de California, 523-526.

Hidalgo Navarro, Antonio, y Mercedes Quilis Merín, 2004: Fonética y fonología españolas, Valencia: Tirant lo Blanch.

Hidalgo Navarro, Antonio, y Mercedes Quilis Merín, 2012: La voz del lenguaje: fonética y fonología del español, Valencia: Tirant lo Blanch.

HiRaYama, Manami, 2003: "Contrast in Japanese vowels", Toronto Working Papers in Linguistics 20, $115-132$.

Hualde, José Ignacio, 2005: The sounds of Spanish, Cambridge: University of Cambridge. IribarRen, Mary C., 2005: Fonética y fonología españolas, Madrid: Síntesis. 
Ito, Chiyuki, Yoonjung Kang y Michael Kenstowicz, 2006: "The Adaptation of Japanese Loanwords into Korean", MIT Working Papers in Linguistics 52, 65-104.

Itô, Junko, y Armin Mester, 1995: "Japanese Phonology. Constraint Domains and Structure Preservation” en John Goldsmith (ed.): A Handbook of Phonological Theory, Oxford, Reino Unido: Blackwell Handbooks in Linguistics Series, 1-23.

Japan Travel Bureau [jtb], 1991 [1989]: Illustrated Japanese Characters, Japón: Japan Travel Bureau.

KanEKo, Emiko, 2006: "Vowel selection in Japanese on-line adaptation of English words", LSO Working Papers in Linguistics (Proceedings of WiGL 2006), Madison: Linguistics Student Organization, University of Wisconsin-Madison.

Kıм, Joe Eun, 2007; “Evidence of /I/-/r/ contrast in Korean”, Saarbrücken, 6-10.

Kondo, Mariko, 2005: "Syllable structure and its acoustic effects on vowels in devoicing environments" en Jeroen van de WeIJer, K. Nanjo y T. Nishihara (coords.): Voicing in Japanese, Berlín: Mouton de Gruyter, 229-245.

Kubozono, Haruo, Junko Itô y Armin Mester, 2009: "Consonant Gemination in Japanese Loanword Phonology" en The Linguistic Society of Korea (ed.): Current Issues in Unity and Diversity of Languages. Collection of Papers Selected from the 18th International Congress of Linguists [CIL 18], República de Korea: Dongam Publishing Co., 953-973.

LABRUne, Laurence, 2012: The Phonology of Japanese, Oxford: Oxford University Press.

Ladefoged, Peter, y Keith Johnson, 2011 [1975]: A course in phonetics, Boston: Wadsworth/Cengage Learning.

Ladefoged, Peter, e lan Maddieson, 1996: The Sounds of the World's Languages, Oxford: Blackwell.

LAPESA, Rafael, 2005 [1981]: Historia de la lengua española, novena edición, Madrid: Gredos.

LEVITT, June S., y William F. KATZ, 2008: "Augmented visual feedback in second language learning: Training Japanese post-alveolar flaps to American English speakers", POMA (2), Universidad de Texas, 1-13.

MaEkAWA, Kikuo, 2003: "Corpus of Spontaneous Japanese: its design and evaluation”, Proceedings of ISCA and IEEE Workshop on Spontaneous Speech Processing and Recognition (SSPR2003), Tokio, 7-12.

Magnuson, Thomas, 2008: "What/r/ Sounds Like in Kansai Japanese: A Phonetic Investigation of Liquid Variation in Unscripted Discourse". Tesis de doctorado, University of Victoria: Canadá [http://dspace.library.uvic.ca:8080/bitstream/handle/1828/1367/Magnuson_08MA_ThesisKansai_ns_R.pdf?sequence=1, fecha de consulta: 27 de mayo de 2016]. 
Magnuson, Thomas, 2009: "A pharyngeal component in Kansai Japanese /r/ variants?", comunicación presentada en the International Workshop on Pharyngeals and Pharyngealisation, 26-27 marzo 2009, Newcastle upon Tyne: Reino Unido.

Magnuson, Thomas, 2011: "Realizations of / $r$ / in Japanese talk-in-interaction", comunicación presentada en 17th International Congress of the Phonetic Sciences, 17-21 agosto 2011, Hong Kong, 1306-1309.

Martinet, André, 1974: Economía de los cambios fonéticos, Madrid: Gredos.

MartinezCeldrán, Eugenio, 1989: Fonologíageneralyespañola:fonologíafuncional, Barcelona:Teide.

Martínez Celdrán, Eugenio, 1996: “Evaluación de los cuadros de fonemas”, Lingüística Española Actual 18 (1), 5-16.

Martínez Celdrán, Eugenio, 2000: "Fonología funcional del español” en Manuel Alvar (dir.): Introducción a la Lingüística española, Barcelona: Ariel, 139-153.

Martínez Celdran, Eugenio, y Ana M. ${ }^{a}$ Fernandez Planas, 2007: Manual de Fonética española: articulaciones y sonidos del español, Barcelona: Ariel.

MatsuUra, Yunichi, y Lourdes Porta Fuentes, 2000: Japonés para hispanohablantes. Gramática, Barcelona: Herder.

Moliner, María, 2008 [1996]: Diccionario de uso del español, tercera edición, Madrid: Gredos [CD-ROM].

Navarro Tomás, Tomás, 2004 [1918]: Manual de pronunciación española, Madrid: C.S.I.C.

Nogita, Akitsugu, 2010: "Examination of the [si] and [ $\mathrm{i}$ ] Confusion by Japanese ESL Learners". Tesis de doctorado, 2010, University of Victoria: Canadá [https://dspace.library.uvic.ca:8443/ bitstream/handle/1828/2991/UVic\%20MA\%20thess\%20kitsugu\%20Nogita.pdf?sequence=1, fecha de consulta: 27 de mayo de 2016].

OнатA, Kota, 2004: "Phonological Differences between Japanese and English: Several PotentiaIly Problematic Areas of Pronunciation for Japanese ESL/EFL Learners”, Asian EFL Journal 6 (4).

OkadA, Hideo, 1999: "Japanese”, Handbook of the International Phonetic Association: A guide to the usage of the International Phonetic Alphabet, Cambridge: Cambridge University Press, 117-119.

Quilıs, Antonio, 2006 [1993]: Tratado de fonología y fonética españolas, Madrid: Gredos.

Real Academia Española [RAE], 2010: Ortografía de la lengua española, Madrid: Espasa.

Real Academia Española [RAE], 2011: Nueva gramática de la lengua española: fonética y fonología, Barcelona: Espasa. 
Real Academia Española [RAE], 2014: Diccionario de la Real Academia Española, Madrid: Espasa Libros [http://www.rae.es/, fecha de consulta: 27 de mayo de 2016].

Rodriguez-IzQuierdo y Gavala, Fernando, 1991: "Sobre los sistemas de Transliteración alfabética del japonés, y sus posibles adaptaciones destinadas a hispanohablantes", Boletín de la Asociación Española de Orientalistas, año 27, 121-130.

SAIto, Akemi, 2005: "Análisis de errores en la expresión escrita de los estudiantes japoneses". Tesis de maestría, Universidad de Salamanca [http://www.mecd.gob.es/dctm/redele/MaterialRedEle/Biblioteca/2005_BV_03/2005_V_03_13Saito.pdf?documentld=0901e72b80e40236, fecha de consulta: 27 de mayo de 2016].

SAlvador, Gregorio, y Juan Ramón Lodares, 2008 [1996]: Historia de las letras, Madrid: Espasa Calpe. SÁnChez, Aquilino, 2001 [2006]: Gran diccionario de uso del español actual, Madrid:SGEL [CD-ROM]. Seco, Manuel, Olimpia Andrés y Gabino Ramos, 1999: Diccionario del español actual, Madrid: Aguilar.

SeEley, Christopher, 2000 [1991]: A history of writing in Japan, University of Hawai'i Press.

Shibatanı, Masayoshi, 1990: The Languages of Japan, Cambridge: Cambridge University Press.

SмIтH, R. Edward, 1980: "Natural Phonology of Japanese". Tesis de doctorado, Universidad de Hawaii [http://www.trussel.com/jap/edsmith.htm, fecha de consulta: 27 de mayo de 2016].

Teshigawara, Mihoko, 2002: "Vowel Devoicing in Tokyo Japanese" en Geoffrey Stewart Morrison y Les Zsoldos (eds.): Proceedings of the North West Linguistics Conference, Canadá: Simon Fraser University Linguistics Graduate Student Association, 61-66.

Trubetzkoy, Nikolai S., 1973 [1939]: Principios de fonología, Madrid: Cincel.

Tsujımura, Natsuko, 2007 [1996]: An Introduction to Japanese Linguistics, Malden, MA: Blackwell Publishing.

UedA, Hiroto, 1977: "Estudio contrastivo de los sonidos españoles y japoneses (1): vocales y semivocales”, Lexicon 6, 29-46.

Unger, Marshall, 2003 [1996]: Literacy and Script Reform in Occupation Japan: Reading Between the Lines, Oxford: Oxford University Press.

Vance, Timothy J., 1987: An Introduction to Japanese Phonology, State University of New: New York Press.

VANCE, 2008: The Sounds of Japanese, Cambridge: Cambridge University Press. 